Virtual and interactive 3D-vascular-reconstruction before planned pancreatic head resection and complex vascular anatomy: A bench-to-bedside transfer of virtual reality

(Abstract ID: 279)

R. Templin ${ }^{1}$, D. Weyhe ${ }^{1}$

${ }^{1}$ Pius-Hospital Oldenburg

\title{
Background:
}

Bühlers' anastomosis (also known as Bühlers' arcade) is an embryonic relic and represents an arterioarterial connection between the superior mesenteric artery and the celiac trunk. It can be found as a variety in $1-2 \%$ of patients.

\section{Materials and methods:}

We present a case in which a patient with metatastatic squamous cell carcinoma of the lung has been in stable disease for 4 years under palliative therapy (most recently second-line therapy with Nevolumab). In 2019, a locally advanced adenocarcinoma of the papilla vateri was also diagnosed. The patient also had to undergo right hemicolectomy and patch plasty of the celiac trunk and superior mesenteric artery due to colonic ischemia and arteriosclerotic disease with stenosis of the superior mesenteric artery several years ago. For the planning of a pylorus-preserving pancreatic head resection and reconstruction according to Traverso-Longmire, the preoperative imaging was extended by two independent vascular reconstructions due to a complex vascular prehistory.

\section{Results:}

The Bühler anastomosis was detected in an digital, virtual and interactive 3D-reconstruction. In addition, a 3D print of the abdominal site was made. Both was created as an off-label use as a part of a research project in cooperation with the TZI of the University of Bremen and the Frauenhoferinstitut (VIVATOP: Versatile Immersive Virtual and Augmented Tangible OP). In the actual clinical CTangiography, this variant had not been described. Intraoperatively, the presence of Bühler's anastomosis was confirmed. However, this information had a significant impact on the intraoperative approach. Retrospectively, this vessel variant could be surmised in the axial projection of the CT scan.

\section{Conclusion:}

Increasing digitalization in surgical and perioperative preparation, in addition to its special requirements, holds great potential for better planning and improved patient safety. Research and cooperation projects such as the VIVA TOP project are indispensable for this. 


\section{Picture:}
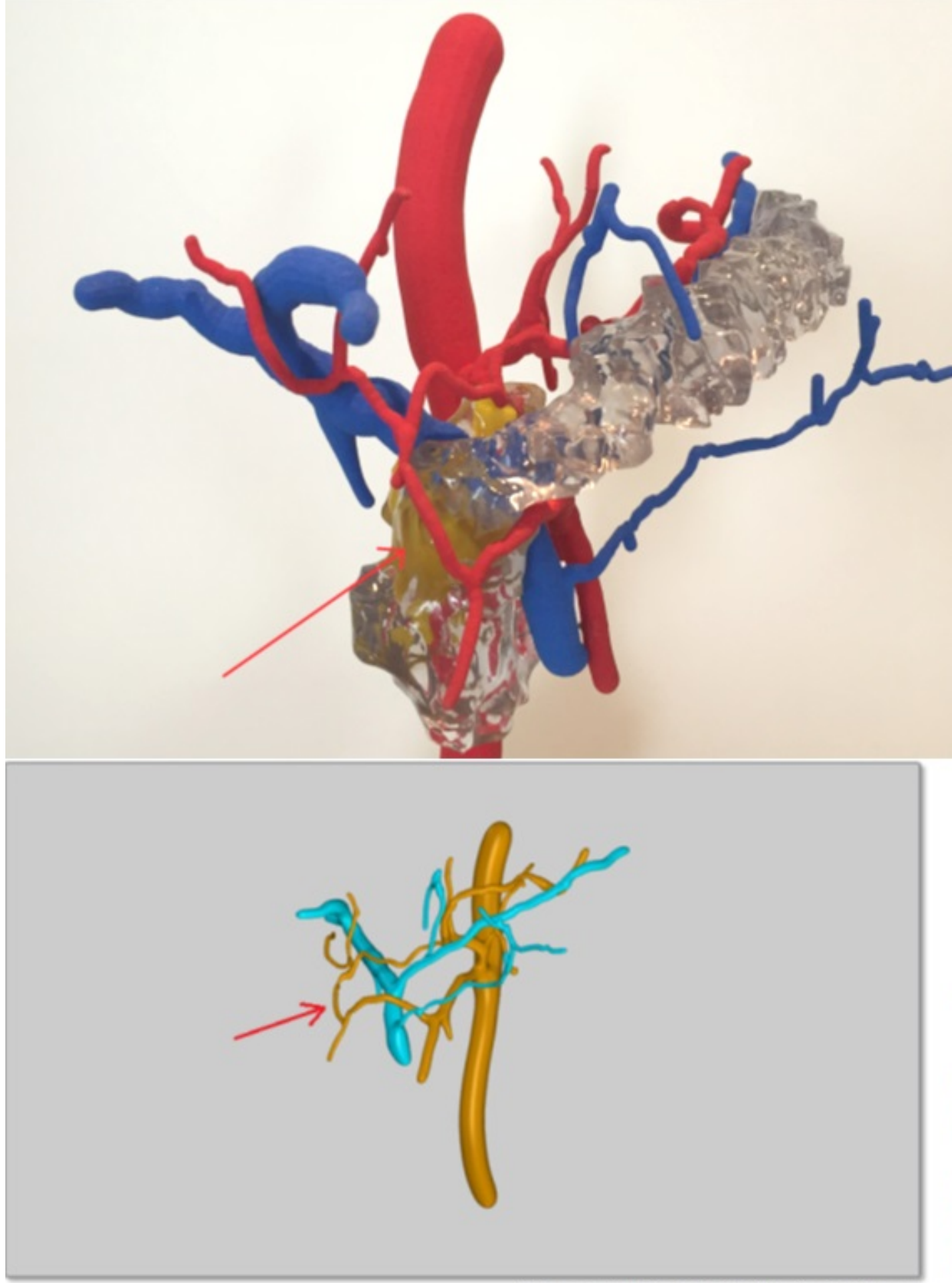


\section{Robotic-assisted major liver resection - technical aspects}

(Abstract ID: 332)

J. Pratschke ${ }^{1}$, W. Schöning ${ }^{1}$, M. Schmelzle ${ }^{1}$

${ }^{1}$ Charité - Universitätsmedizin Berlin - CVK

\section{Background:}

A structured program for the development of robotic-assisted liver surgery was established at our department in 2019. We here report on experiences gained during the course of twenty major liver resections with the Da Vinci Xi Surgical System (Intuitive Surgical Inc.).

\section{Materials and methods:}

We here present a representative video of left-sided robotic assisted hemihepatectomy in in a patients with a biliary cystadenoma. Presented intraoperative strategies and techniques represent the current standard in robotic-assisted major liver resections at our center.

\section{Results:}

We report on a 36 -year old patient with an $85 \mathrm{~mm}$ large biliary cystadenoma of the left liver lobe. A robotic-assisted left hemihepatectomy was performed using the Da Vinci Xi Surgical System. The operation was performed in 7-trocar technique with four $8 \mathrm{~mm}$ Da Vinci trocars, one $5 \mathrm{~mm}$ and two $12 \mathrm{~mm}$ assist trocars. After mobilization of the left lobe of the liver, the left hepatic artery and the left portal branch were selectively displayed and transected between clips. Under repetitive sonographic control, the parenchymal dissection was performed using an ultrasound scissor. Crossing intraparenchymal structures were selectively displayed and transected. The left hepatic duct was visualized at an early stage and selectively transected between clips. The parenchymal transection was performed in the sense of a caudal approach with the transection of the left hepatic as the last step. The specimen was retrieved in a bag via a median transumbilical mini-incision. The operation time was 4.59 hours. There were no postoperative complications, allowing to discharge the patient on postoperative day 6 .

\section{Conclusion:}

Experiences made in complex laparoscopic liver surgery can be safely translated to robotic-assisted liver surgery. Possible advantages of robotic-assisted liver surgery over laparoscopic surgery are most evident in the selective preparation of lymph nodes and hilar structures. 
Serious Gaming and Virtual Reality in the multimodal training of minimally invasive surgery: A randomized crossover assessment and validation of laparoscopic inguinal hernia repair training

(Abstract ID: 807)

\author{
E. Willuth ${ }^{1}$, F. Lang ${ }^{1}$, C. Haney ${ }^{1}$, E. A. Felinska ${ }^{1}$, K.-F. Kowalewski ${ }^{1}$, M. W. Schmidt ${ }^{1}$, B. P. Müller- \\ Stich $^{1}$, F. Nickel \\ ${ }^{1}$ Chirurgische Klinik, Universitätsklinikum Heidelberg
}

\title{
Background:
}

For a good surgical outcome, specific knowledge about the anatomy and the individual steps of operations are indispensable. New learning materials are constantly being developed for this purpose and to ensure the best possible training for surgeons. The research in surgical teaching examines the effectiveness and benefits of these multimodal training settings. The aim of this study was to assess the transferability of surgical skills between the laparoscopic hernia module on the serious game TouchSurgeryTM(TS) and on the virtual reality (VR) trainer LapMentorTM. Additionally, a validation of the laparoscopic hernia repair on TS is foreseen.

\section{Materials and methods:}

This was a prospective monocentric randomized cross-over study in the training center of minimally invasive surgery. It was conducted within a voluntary elective module for medical students in their 3rd to 6 th year of medical school at Heidelberg University $(n=40)$. All participants were randomized in a 1:1 ratio and stratified by gender to group 1 and group 2. Group 1 started with the "Laparoscopic Inguinal Hernia Module" on TS (phase 1: patient preparation, phase 2: hernia repair) and performed the module first in training mode, then in test mode until proficiency goals were reached. Group 2 started with the "Inguinal Hernia Module" on a VR trainer (task 1: anatomical identification, task 2: incision and dissection) and also performed the module until proficiency goals were reached. Once the goals reached, the groups changed and performed the other training modality until proficiency goals were reached. Primary endpoints were the number of attempts needed to achieve the goals for each group and for each task/phase on both tools. The outcomes were statistically tested using a chisquared test. P-value under 0.05 was deemed to be significant.

\section{Results:}

Students who performed on TS first needed significantly less attempts to reach the predefined goal for task 1 on the VR trainer than students who directly started with the VR trainer (TS $=2.70 \pm 0.57$ vs VR $=3.16 \pm 0.69 ; p=0.031$ ). No significant differences for task 2 were observed between the groups (TS $=2.25 \pm 1.12$ vs $V R=2.05 \pm 0.85 ; p=0.537$ ). For both phases on TS no significant skill transfer from the VR trainer to TS was observed.

\section{Conclusion:}

The results show that surgical skills could be transferred from TS to the VR trainer for task 1 but not for task 2. In fact, TS provides only theoretical training and task 1 tests anatomical knowledge, task 2 tests practical skills. Skill transfer from the VR trainer to TS as we expected could not be shown, as the module on the VR trainer teaches purely practical knowledge about the procedure. Also, the VR trainer teaches only the peritoneal preparation (net placement is still under development) and does not teach patient preparation. VR and TS should thus be used in combination in multimodal training to ensure optimal training conditions. 


\title{
Clinical und functional outcome following robotic myotomy with partial fundoplication in patients with achalasia
}

(Abstract ID: 1000)

\author{
M. S. Rabe ${ }^{1}$, B. Jansen-Winkeln ${ }^{1}$, M. Mehdorn ${ }^{1}$, D. Buchloh', O. Lyros ${ }^{1}$, I. Gockel ${ }^{1}$, S. Niebisch ${ }^{1}$ \\ ${ }^{1}$ Universitätsklinikum Leipzig AöR
}

\section{Background:}

The minimal invasive myotomy with partial fundoplication for symptomatic patients with achalasia is the gold-standard. It has been established as a safe and effective procedure in the past decades. With the rise of robotic-assisted surgery in recent years, studies comparing both procedures have shown similar short-term results with a lower rate of intraoperative esophageal perforations in patients treated robotically. However, due to a lack of long-term results providing clinical and functional outcomes, our aim was to investigate a collective undergoing robotic-assisted surgery providing pre- and postoperative established symptom score und high-resolution manometry.

\section{Materials and methods:}

All patients were underwent clinical, endoscopic, radiological und manometric investigation to verify the diagnosis of achalasia. High-resolution manometry was performed preoperatively und 6 months following surgery and was categorized according to the current Chicago-Classification (v3.0). Furthermore, we used the Eckhardt Score to evaluate symptomatic outcome. All patients underwent robotic-assisted myotomy with partial anterior fundoplication using the DaVinci Xi surgical system (Intuitive, Sunnyvale, California, USA).

\section{Results:}

We analyzed 35 consecutive robotic procedures for achalasia between September 2017 and September 2019. Included were 18 male and 17 female patients with median age of 48 years. All patients showed a significant improvement of symptoms subjectively with a significant decrease of the Eckhard score from 8 preoperatively to 3 postoperatively. The postoperative lower esophageal resting pressure (LESP) and residual pressure (4s-IRP) was significantly reduced in all patients (median LESP $40,6 \mathrm{mmHg}$ vs. $11,9 \mathrm{mmHg}$; median $4 \mathrm{~s}-$ IRP $29,6 \mathrm{mmHg}$ vs $10 \mathrm{mmHg}$ ). There was no event of intraoperative esophageal perforation or conversion to open surgery.

\section{Conclusion:}

Our data support the feasibility and effective robotic approach for the surgical treatment of achalasia. Although, our data are based on a short-term follow up, our results are non-inferior to the reported laparoscopic approach. Further investigations in larger prospective multicenter studies are needed to verify a potential superiority of the robotic approach. 


\title{
A new tool in battling anastomotic leak: Objective intraoperative perfusion assessment in real- time during complex abdominal surgery - a pilot study of colon interpositions
}

\author{
(Abstract ID: 837)

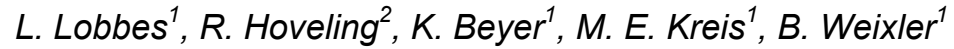 \\ ${ }^{1}$ Charité Universitätsmedizin Berlin (CBF) \\ ${ }^{2}$ Quest Medical Imaging, Middenmeer
}

\section{Background:}

Anastomotic leak is a common complication after esophageal reconstruction with colon interposition in critically ill esophageal cancer patients. Ischemia as well as decreased microperfusion contribute significantly to this complication. Up to date, surgeons have to rely solely on clinical signs of ischemia when assessing colon graft perfusion intraoperatively. Near-infrared (NIR) fluorescent imaging with indocyanine green (ICG) could help in assessing intestinal perfusion objectively as during the procedure there is only limited information on impaired perfusion available to the surgeon. We investigated a novel software for NIR tissue perfusion imaging in esophageal cancer patients undergoing colon interposition surgery.

\section{Materials and methods:}

Three patients with esophageal cancer who underwent colon interposition for esophageal reconstruction in 2019 were included. Patients 1 and 2 each received an isoperistaltic left colonic interposition, patient 3 received an anisoperistaltic left colonic interposition.

Tissue perfusion of the colon graft and the respective anastomoses was measured intraoperatively at three different time points with the Quest Spectrum® (Quest Medical Imaging, Middenmeer), a device for fluorescence guided surgery. At time point one after isolation of the colon graft) a dose of $5 \mathrm{mg}$ ICG was administered intravenously. At time point two (after construction of the cervical anastomosis) again a dose of $5 \mathrm{mg}$ ICG was administered intravenously. At time point three (after contruction of the abdominal anastomosis) a dose of $20 \mathrm{mg}$ methylene blue was administered intravenously.

During the administration of ICG and methylene blue, images were acquired for 90 seconds at the mentioned locations with the Quest Spectrum $®$ to register the changes of the NIR fluorescence signal over time. Postoperative analysis of the signal changes was performed with the Quest Research Software for different regions of interest (ROI). ROls where selected in the areas that were planned for anastomosis and for the oral and aboral side of the anastomosis in the cervical and abdominal region. Changes in the NIR-fluorescence signal were plotted in a graph to get an insight of the ingress and egress phase of the tissue perfusion.

\section{Results:}

Three patients (two male) were included in this pilot study. Patient 1 developed anastomotic microleak of the cervical site at postoperative day 7 . In patient 2 the colon graft failed at postoperative day 5 with anastomotic leak at both sides. In patient 3 (female) no anastomotic leak occured. The tissue perfusion graphs for patient 1 show a deviation in ingress and egress in the region selected for the failed cervical anastomosis compared to the successful distal anastomosis in all 3 measurements. For patient 2 the perfusion graphs for the colon graft as well as for the colonic side of the cervical and abdominal anastomoses show low ingress rates and positive egress rates, which are comparable to the graphs observed for the failed anastomosis in patient 1 . In the graphs of all measured phases of patient 3 perfusion graphs were observed with similar ingress and egress patterns as shown in the abdominal anastomosis region of patient 1 . 


\section{Conclusion:}

Postoperative evaluation of our intraoperative imaging data shows that a significant decrease in perfusion was already detectable in the area of future anastomotic leak intraoperatively with the aid of the software used here for the first time worldwide. Further studies on more patients are needed to elucidate the clinical value of this novel technique.

\section{Picture:}

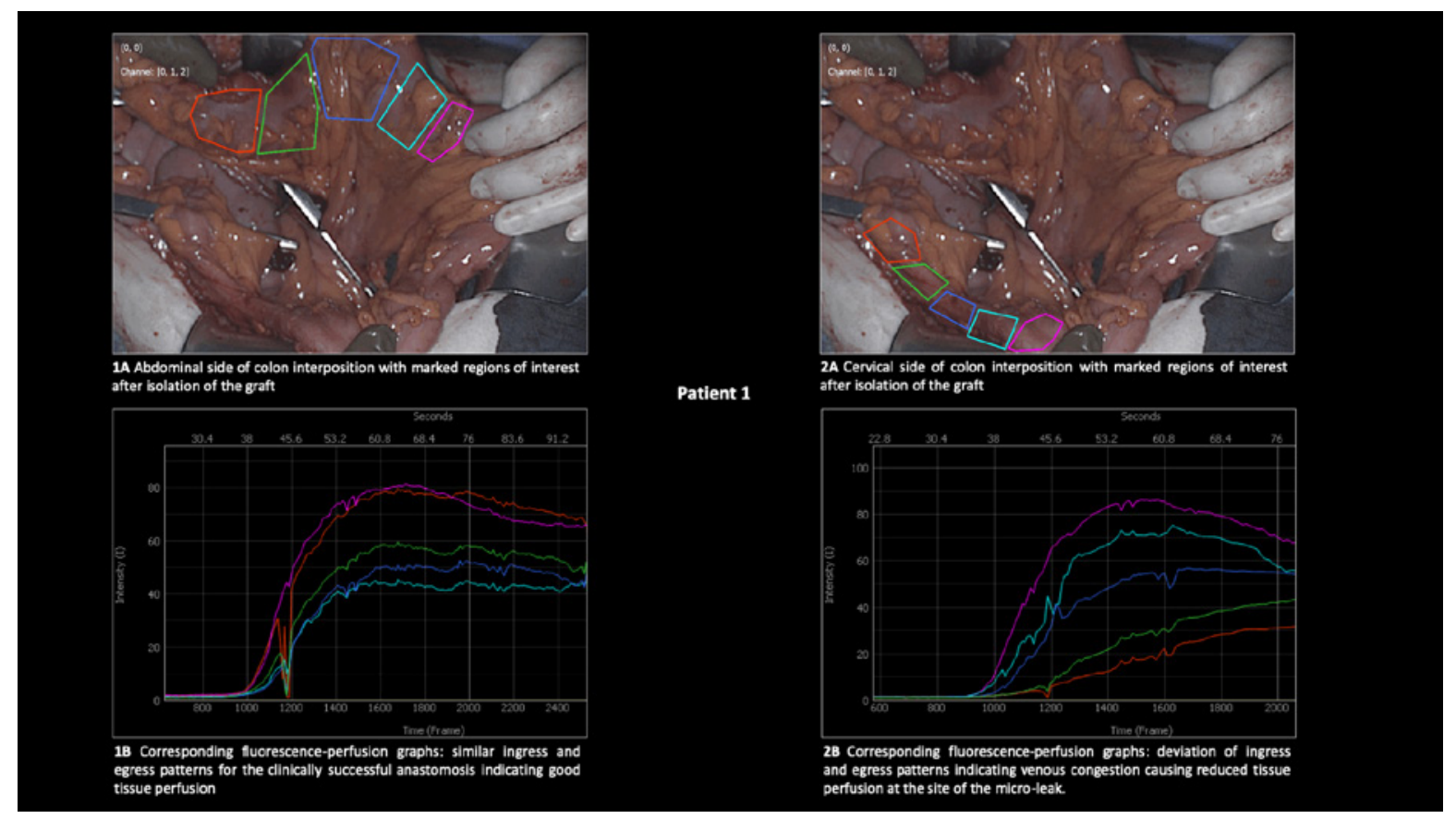




\title{
How I do it: Animal models in Vascularized Composite Allotransplantation research: Current Concepts
}

(Abstract ID: 1023)

\author{
A. Dermietzel ${ }^{1}$, M. Aitzetmüller ${ }^{1}$, K. Harati ${ }^{1}$, P. Wiebringhaus ${ }^{1}$, T. Hirsch ${ }^{1}$, M. Kückelhaus ${ }^{1}$ \\ ${ }^{1}$ Universitätsklinikum Münster
}

\section{Background:}

Pre-clinical studies are obligatory tools to implement novel operative, diagnostic as well as therapeutic strategies into clinical workaday life. In the field of transplantation, animal models have proven to be indispensable for improvement of survival and quality of transplants. These models offer access not only to target problems concerning immunological and physiological perspectives but also from technical and surgical points of view. For the uprising field of vascularized composite allotransplantations (VCA) animal models have been essential and found to be crucial for successful clinical application.

\section{Materials and methods:}

For this review a thorough literature search on pubmed was performed in October of 2019 with the following keywords: vascularized composite allotransplantation, vascularized composite allograft, limb transplant, composite tissue allograft / allotransplantation, facial transplant, experimental transplantation, mouse, murine, rat, rabbit, rodent, porcine, pig, swine, dog, canine and primate.

\section{Objective:}

This review displaces a comprehensive overview of the currently most commonly used models of experimental VCA research and the amendments achieved when applied to clinical work. It is intended to outline the pros and cons of each model and to help find a suitable protocol for each individual scientific interest.

\section{Results:}

Advantages like low costs, low efforts for conducting experiments and precise genetically breeding are obvious benefits of some of the small animal models. Nevertheless they may face practical disadvantages in terms of anatomical differences to larger animals and microsurgical skill needed, prolonging the learning curve and number of animals needed.

Various approaches with rat models for vascularized composite allotransplantation have been made in the past decades and play a major role as a models for microsurgical training and research in this field. Their smaller but yet surgically doable size proofed to be quite beneficial and cheap for transplantation research.

Porcine large animal models are of great value for translational research of VCA. Due to the larger anatomical proportions and similarities in immune systems, data is more reliable to transfer to humans. Nonetheless large animal models demand an elaborated infrastructure and teams dedicated to conduct this research. Overall the field of interest, time and infrastructure available are important key factors for each researcher to find the perfect model. 


\section{Mentor-mentee relationship in surgical education - an answer from a student perspective}

(Abstract ID: 324)

T. Klemm ${ }^{1}$, F. Loos ${ }^{2}$, S. F. Hertling ${ }^{1}$

${ }^{1}$ Waldkliniken Eisenberg

${ }^{2}$ Gelenkzentrum Leipzig

\section{Background:}

The shortage of doctors is a topical issue. Already today many young doctors are missing. Surgical subjects are particularly affected. The disciplines know about this highly topical subject and try to create incentives with special support programs to inspire young colleagues for their field. Special mentoring mentee programs are offered here. What possibilities do surgical subjects offer? Is there a need for it? Are these programs used? Can a mentoring program positively influence the choice for a surgical subject?This study aim is to provide an answer from a student perspective.

\section{Materials and methods:}

A specific online questionnaire for medical students was created, which is answered anonymously. 8 items are included here: status, gender, participation in mentee mentoring program, subspecialization, benefit, referral, positive impact on your own surgical training, negative experience. A total of 1000 answered questionnaires are included.

\section{Results:}

The results will be presented in this congress. In general it can be said that students are offered a variety of mentee mentoring programs and that these are used to an above- average level. Students benefit from an organized mentoring program during their medical education and they positively influence the choice for a surgical subject. Students who already had a mentor in a surgical field are more likely to opt for a surgical doctor's education.

\section{Conclusion:}

Mentee Mentoren programs seem to have a positive effect on surgical training. They should become an integral part of programs to improve the recruitment of young doctors in surgical subjects and to support their career development and productivity in research. Mentoring should also be offered to students. 
CUT - a surgical-anatomical project for medical students, based on interactive, innovative and interdisciplinary teaching methods

(Abstract ID: 952)

F. Lang ${ }^{1}$, E. Felinska ${ }^{1}$, J. Ito ${ }^{1}$, J. Rom ${ }^{2}$, B. P. Müller-Stich ${ }^{1}$, J. Kirsch ${ }^{3}$, F. Nickel ${ }^{1}$

${ }^{1}$ Universitätsklinikum Heidelberg

${ }^{2}$ Klinikum Frankfurt Höchst, Frankfurt am Main

${ }^{3}$ Universität Heidelberg

\section{Background:}

"CUT - ChirUrgische AnaTomie" is a unique series of events for medical students in all semesters. CUT represents an interdisciplinary cooperation of the surgical department, the institute of Anatomy and Cell Biology together with the student council of medicine of the University of Heidelberg. The elective course connects classical and innovative educational approaches with the latest digital media and technologies, thereby promoting the integration of digital teaching and traditional hands-on learning concepts in surgical education.

\section{Materials and methods:}

During CUT, experienced surgeons perform live operations on body donors together with medical students and anatomy specialists in front of 40-80 medical students on three consecutive days. Interactive teaching of anatomy is complemented by surgical demonstration of the anatomical field and surgical steps. Students have the opportunity of active participation in the operations by means of regular rotation, thus attending a direct hands-on experience on the surgical table. This offers participants the possibility to further develop their surgical skills and to perform particular operation steps themselves under direct supervision. Simultaneously, a digital multimedia transmission occurs for the participating students with different camera perspectives and digital means of interactions that are evaluated for their added value to the teaching event. Participants take active parts in preparation of the event and evaluation of educational concepts. Moreover, students deepen their knowledge of anatomy and surgery by giving talks and presenting studies on the surgeries performed.

\section{Results:}

Evaluations, which have been regularly performed during the event, show that a total of $96 \%$ of more than 400 participants so far are "very enthusiastic" about the whole concept of the CUT event and appreciate the innovative character of the organization. Especially the applied technologies, such as augmented reality, digital laser pointer and three-dimensional virtual anatomy have been rated positively in every way. In particular, the usage of computed tomography (CT) scans that have been made from the body donors was rated equally positive by the participants.

\section{Conclusion:}

The integrated teaching approach that is applied in the CUT event series greatly motivates active learning. Medical students benefit from the practical application of anatomical knowledge that has been previously learned in the preclinical period and can now be transferred to clinical subjects. Moreover, active participation in surgical procedures in a realistic setting that resembles the operating room helps to strengthen acquired skills and to close remaining gaps. An interactive multimedia learning concept that integrates virtual reality, augmented reality, hyperrealism and modern video recording improves surgical teaching processes and medical training. Additionally, students have the opportunity to enhance their presentation skills in an academic environment and can benefit from 
modern teaching approaches, such as learning by teaching, multi-sensual learning and manifold learning methods. Besides promotion of the professional expertise and further development of students' soft skills, the CUT events provide an excellent opportunity for recruiting young surgical talents. 


\section{How to deal with senescent patients in the field of visceral surgery?}

(Abstract ID: 949)

H.-M. Tautenhahn ${ }^{1}$, F. Dondorf ${ }^{1}$, P. Felgendreff , A. Bauschke ${ }^{1}$, U. Dahmen ${ }^{1}$, F. Rauchfuß ${ }^{1}$, U. Settmacher ${ }^{1}$

${ }^{1}$ University of Jena Medical Center

\section{Background:}

Senescence is considered a risk factor for major surgery. Due to a lack of scientific evidence, diagnostic and therapeutic decisions are often based on the clinical experience of the individual surgeon. The Center of Geronto-Surgery Thuringia was founded to improve the outcome after major surgery for this growing group of patients (pts) at risk. To better characterize the age-dependent risk profile, we performed a retrospective analysis of the in-hospital results after extended visceral resection (EVR) performed in the last 5 years $(y)$.

\section{Materials and methods:}

Patients from the Department of General, Visceral and Vascular Surgery subjected to EVR in the time period from 2013 on and either below 35 or above 65 years were included in the analysis. Using the clinic patient information system all clinically relevant parameters (e.g. age, sex, comorbidities, duration of hospital stay, complication rate...) were extracted and imported into an Access database. Statistical analyses were performed using $\mathrm{R}$.

\section{Results:}

In the study period of seven years over 17,000 old patient cases were included. The absolute and relative number of old patients increased significantly over the years. In The postoperative morbidity in terms of such as cardiovascular, pulmonary or neurological complications was significantly higher in the geriatric group compared to the young ( $x \%$ versus $y \%$ ), corresponding to the significantly longer hospital stay( a days versus $b$ days, $p<0.05$ ). However, no difference was detected in the $30 \mathrm{~d}$ mortality. The length of hospital stay also was significantly longer in the geriatric patients.

\section{Conclusion:}

Despite the increasing number of senescent patients subjected to EVR and the significantly higher postoperative morbidity, no special pre-, peri- and postoperative treatment protocols are available for this population at risk. Since excluding this population from EVR is ethically not justified, age-adapted peri-operative strategies need to be developed. Even better is the establishment of multi-disciplinary geriatric-surgery centers with a special focus on the special needs of this group of patients in need. 


\title{
Evaluation of quality and sustainability of an outpatient, telemedical care project in a surgical university clinic based on a PDCA checklist
}

\author{
(Abstract ID: 59) \\ J. Gödeke ${ }^{1}$, O. Muensterer ${ }^{1}$ \\ ${ }^{1}$ Universitätsmedizin Mainz
}

\section{Background:}

Germany is still in a kind of "telemedical finding phase" in 2019. Regularly existing telehealth video consultations in the field of surgery are still the exception and they are usually associated with research projects, e.g. linked to the Innovation Fund of the Federal Joint Committee, as the operating costs to date can only be redeemed inadequately beyond the general possibilities. Selective contracts with individual health insurances can be one way of compensating for telemedical video consultation in a cost-covering and equitable manner, thus consolidating the telemedical approach. For this, however, numerous quality and sustainability criteria must necessarily be met, which are not yet published in structured form for surgical telemedicine consultation. The aim was therefore to develop a checklist with which the most important quality and sustainability criteria can be queried in a standardized way in a straightforward manner.

\section{Materials and methods:}

In an initial survey, two statutory health insurance companies were asked about their personal requirements for concluding a direct contract with a medical service provider. As part of an expert survey and current literature, a checklist for identifying and evaluating the quality and sustainability potential of a clinical telemedicine care project by means of video communication was then prepared in the form of the so-called PDCA cycle (Plan / Do / Check / Ad). This was finally applied to the existing own pediatric surgical telemedical platform "MATS" (MAinz TeleSurgery) and evaluated for the first time.

\section{Results:}

The checklist was based on a large number of success factors from the areas of market environment, quality and benefits statement, financing and reimbursement as well as patient needs and consists of 82 criteria, which could be divided into the four action fields PDCA. In the internal evaluation of the own telemedical consultation, the high quality and also the existing sustainability potential, but also valuable weaknesses of the own telemedical consultation were determined. Proposed solutions to address the vulnerabilities have been developed and partially implemented.

\section{Conclusion:}

The PDCA checklist, developed on the basis of expert knowledge and practical experience, is a new practical tool that can be used to systematically evaluate surgical telemedical consultations regarding quality and sustainability potential. An evaluation of further telemedical consultations other than "MATS" must show how valuable the checklist is in practice. It may be necessary to make adjustments to the checklist in the future. Overall, however, we assume that the checklist evaluates the basic requirements for the conclusion of direct contracts with health insurances. In the future, telemedicine surgical video-consulting sessions could be standardized, distributed and adequately remunerated. 


\section{Picture:}

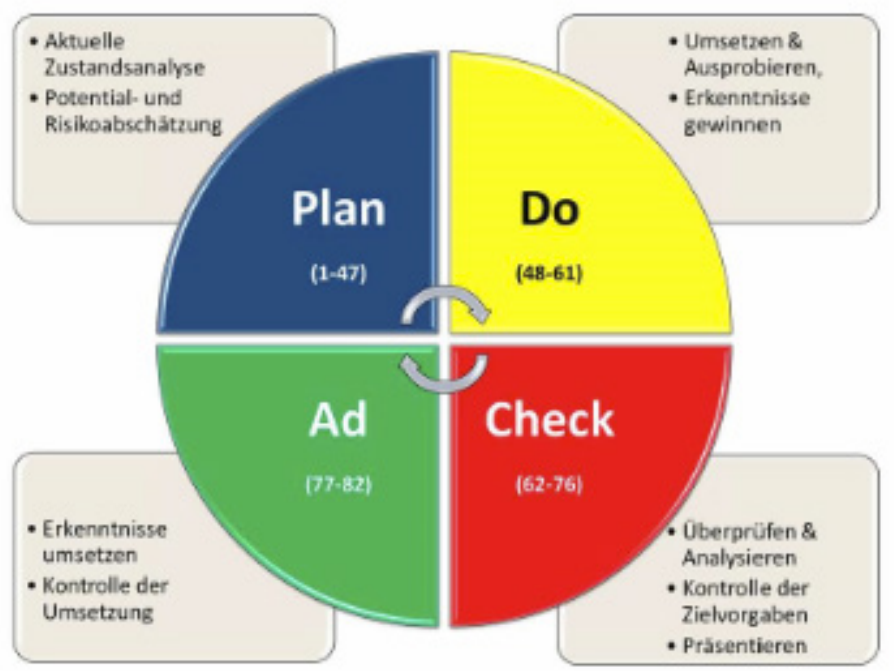

PDCA cycle 


\title{
Assisting the Diagnosis of Left Ventricular Outflow Obstruction and Planning of re-do Surgery with 3D Printing Technology
}

(Abstract ID: 204)

\author{
F. König ${ }^{1}$, S. Abdelaziz ${ }^{2}$, M. Kruttschnitt ${ }^{3}$, N. Hitschrich ${ }^{4}$, B. Mumm ${ }^{4}$, C. Schnell ${ }^{2}$, C. Hagl' ${ }^{1}$, R. Sodian ${ }^{2}$ \\ ${ }^{1}$ Ludwig-Maximilians-University, München \\ ${ }^{2}$ Heart Center Lahr \\ ${ }^{3}$ Technical University of Munich, Garching bei München \\ ${ }^{4}$ Tomtec Imaging Systems, Unterschleißheim
}

\section{Background:}

Left ventricular outflow tract obstruction (LVOTO) is a rare complication of mitral valve surgery. Conventional imaging techniques such as echocardiography, angiography, computed tomography (CT) or magnetic resonance imaging are of excellent diagnostic value. However, in complex clinical situations, 3D-printed models are helpful for decision making and planning of potential non-routine therapies. With this technology, we can help the surgeon or interventional cardiologist by providing a physical model of the anatomy. This allows to better understand and visualize complex anatomical situations and consequently assists in choosing the correct therapeutic actions.

\section{Materials and methods:}

We performed a CT of the patient's chest, visualizing the mitral valve prosthesis and the left ventricular outflow tract (LVOT). The patient's CT dataset was loaded into an image processing and visualization software. We segmented the body of the valve, the left ventricle, the left atrium, the left ventricular outflow tract and the aorta. The 3D models were optimized by removing small free-floating artifacts and by additional polygon mesh fairing.

We then printed the segmented model from white resin. The resulting structure was an anatomically detailed physical model of the patient's heart and the implanted prosthesis.

\section{Results:}

The 3D-printed model allowed a detailed and exact evaluation of the patient's situation. We were able to confirm the rare diagnosis of a narrowed LVOT caused by a strut of a bioprosthetic mitral valve.

Furthermore, this process simplified the preoperative decision making. Cardiologists, surgeons and the patient could see and understand the problem and how to solve it. Moreover, the 3D printed LVOT model helped us to choose the right prosthesis for this patient.

\section{Conclusion:}

In this study, we present the use of a 3D-printed model for diagnosis of a LVOTO after bioprosthetic mitral valve surgery. With a physical model in hand, we were able to visualize the LVOTO and to discuss potential therapeutic options. Moreover, we were able to plan the subsequent re-do surgery in detail using the model. This case shows the immense benefit of 3D printing technologies for surgeons and patients, not only for analysis, but also during the decision-making and pre-operative planning process. 


\section{Picture:}
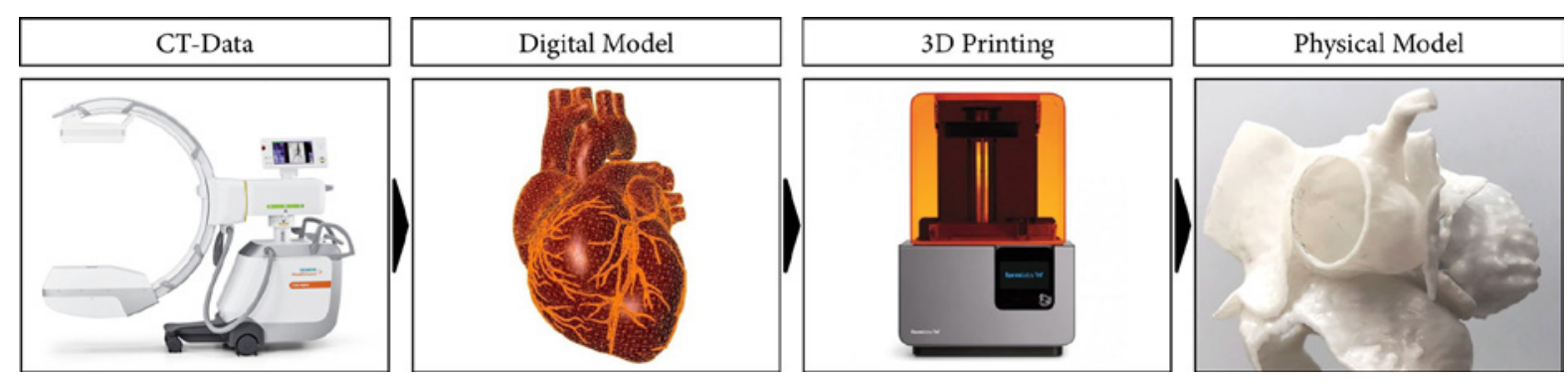
Pectus excavatum: Improved information, diagnosis and care plan by using artificial and collective intelligence

(Abstract ID: 247)

S. B. Sesia ${ }^{1}$, N. Levy ${ }^{2}$, J. Sedbon ${ }^{2}$

${ }^{1}$ Inselspital, Bern University Hospital, University of Bern

${ }^{2}$ Medicalex Francemed, Bagneux

\section{Background:}

Pectus excavatum (PE) is the most common chest wall defect with a frequency of 1:400. Misconception of PE as a 'cosmetic disorder without physiologic consequences' may lead to delayed or no treatment. Therefore, symptoms such as shortness of breath, lack of endurance, or fatigue may worsen with increasing age. Furthermore, treatment of PE at a later stage may be more challenging.

\section{Materials and methods:}

We propose to develop a medical health application (APP) for patients and physicians using artificial and collective intelligence, and aiming to provide early information, diagnosis, and care plan regarding PE. Healthcare data such as biometric data, symptoms, and form, depth and photos of PE is collected adressing the security and privacy requirements.

\section{Results:}

The APP recognizes the type of PE, suggests the most appropriate care plan, monitors the therapy, proposes appropriate adjustments (i.e. use of the suction cup; postoperative consumption of painkillers; assessment of quality of life), and allows statistical analysis for research purposes.

\section{Conclusion:}

Treatment recommendations are based on the use of artificial intelligence. The APP aims to enforce artificial and collective intelligence into a network of stakeholders, to warrant the decision-making for PE patients and specialists, to develop an improved treatment algorithm, and to contribute to a patient registry. 
Leveraging medical imaging for medical education: Photorealistic 3D reconstructions with Cinematic Rendering

(Abstract ID: 469)

C. Krautz ${ }^{1}$, J. Binder ${ }^{1}$, R. Grützmann ${ }^{1}$

${ }^{1}$ Universitätsklinikum Erlangen

\section{Background:}

Cinematic Rendering (CR) is a new physically-based volume rendering technique that may be a beneficial tool for medical education. We aimed to compare $C R$ and conventional computed tomography (CT) imaging for speed and comprehension of anatomy.

\section{Materials and methods:}

This preclinical, randomized, two-sequence crossover study was conducted from July to December 2018, at the University Hospital of Erlangen, Germany. 16 students were randomized into two assessment sequences (CRCT and CTCR). Each participant had to answer 15 anatomy-related questions in both modalities that were divided into three categories: Parenchymal anatomy (PA), musculoskeletal anatomy (MA) and vascular anatomy (VA). After a washout period of 14 days, case evaluations were crossed over to the respective second reconstruction technique. The primary outcome measure was time needed to answer. Secondary outcome was a subjective performance score for each category.

\section{Results:}

Time to answer was significantly shorter for CR assessment. The overall time reduction was $65 \cdot 56 \%$ for all categories. Interperiod difference for MA was $-371 \cdot 3 \pm 65 \cdot 7$ seconds (CRCT) and 396.9 $\pm 77 \cdot 1$ (CTCR), for VA -218.1 $\pm 50 \cdot 1$ (CRCT) and 230.4 $\pm 62 \cdot 7$ (CTCR) and for PA -23.25 $\pm 59 \cdot 9$ (CRCT) and $155.9 \pm 95.5$ (CTCR). The rank order of the performance score was 96 (MA), 95 (VA) and 49 (PA) for $\mathrm{CR}$ and 120 (PA), 76 (VA) and 44 (MA) for conventional imaging. The assessment questionnaire revealed that students consider $\mathrm{CR}$ to be beneficial for medical education and their future professional career. No carryover or period effects were observed.

\section{Conclusion:}

The visualization with $\mathrm{CR}$ allowed a faster comprehension of the radiological anatomy compared to conventional CT imaging. CR has the strong potential to enhance knowledge transfer from medical imaging data and may be used to build up a more customizable study environment. 


\section{Picture:}

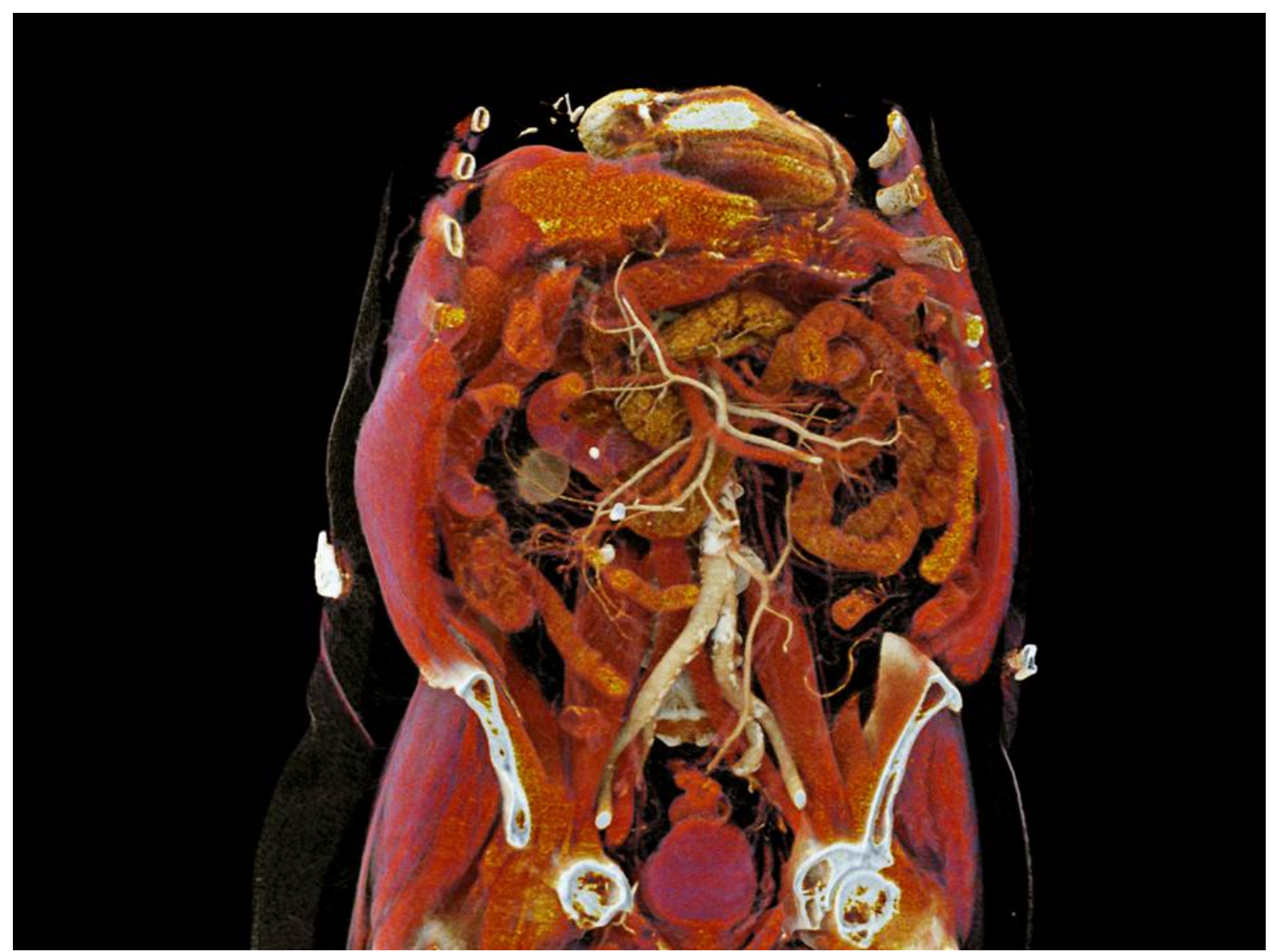




\section{Surgery goes EPA (entrustable professional acitivities) - an irresistible simple app to launch a revolution in assessing clinical skills in medical education}

(Abstract ID: 748)

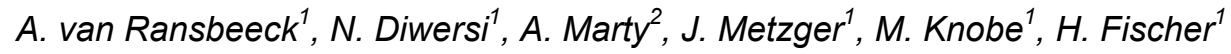

${ }^{1}$ Kantonsspital Luzern

${ }^{2}$ Universitätsspital Zürich

\section{Background:}

Previously used workplace-based assessments (i.e. DOPS, Mini-CEX) are well established and are associated with comprehensive documentation. In every day routine they often are too timeconsuming and therefore performed rarely.

Actual studies point out an insufficient feedback culture and the scarcely performed assessments in medical education. The assessments are usually fragmented into domains like knowledge, clinical skills or communication without evaluating an overall picture.

The concept of "entrustable professional activities" (EPA) is an assessment method specifically wellsuited for daily clinical routine. Focused on an integral assessment an EPA reviews an explicit and observable clinical activity, containing knowledge, skills and professional attitude at a specific moment. The assessment scale is based on the required level of supervision. This scale is already implicitly used by instructors but so far, without the according documentation.

Our study is based on an mobile application installed on trainee's and supervisor's smartphones. All assessment data are collected through the app. The software is most intuitive to operate and convincing in its simplicity. The basic process of an assessment is done with five clicks and takes less than one minute. Every EPA offers five levels of supervision assessed by trainee and supervisor simultaneously. Subsequently the app offers a feedback part and the option to define learning goals. Throughout the course of collecting EPAs, every trainee builds a personalized competency profile. This might serve as a basis for further teaching and promotion.

At the very center is the trainee, she or he has maximum control over all sampled data as data transfer and backup is encrypted.

Hypothesis: Increasing workplace-based assessments and improved feedback culture through a highly user-friendly app.

\section{Materials and methods:}

An existing app, used and tested in departments of anesthesiology in several Swiss clinics, was adapted to surgical medical education. In an iterative process, the authors generated 40 surgical EPAs which correspond with the international quality standards for EPAs (EQUAL).

The study takes place at the Department of surgery, Kantonsspital Luzern, Switzerland. Enclosed are 30 trainees and 20 supervisors. To test the usability of the app only the login data were provided, explicitly without giving further instructions to the users.

During the test phase of 5 months (July to November 2019) monthly feedback rounds about the level of the assessment activity is given.

The impact of the app on the feedback culture is measured through a pre and post interventional questionnaire. Similarly, the user-friendliness is quantified by a final questionnaire. 


\section{Results:}

Data analysis will be completed in December 2019. Aim of the new concept is a simplified and meaningful assessment system with an increased amount of data points. Simultaneously the feedback culture should be affected in a positive way.

True Competency-based Medical Education (CBME) is neither based on the length of the medical education nor the quantity of the performed procedures. At the end only the outcome of a competent doctor is relevant.

Transparent and personal competency profile will optimize the individual training curve of the medical education. Loss of know-how by departmental change is reduced. Supervisors are able to tailor their teaching and supervision specifically to the needs of the trainees. Thanks to the individual competency profiles, promotion and assessment can take place are finally based on data and not only gut feeling. 


\title{
Surgical Skill Assessment Using Machine-Learning Algorithms
}

\author{
(Abstract ID: 476) \\ J. Lavanchy ${ }^{1}$, J. Zindel ${ }^{1}$, K. Kirtac ${ }^{2}$, E. Hosgor ${ }^{2}$, D. Candinas ${ }^{1}$, G. Beldi ${ }^{1}$ \\ ${ }^{1}$ Universitätsklinik für Viszerale Chirurgie und Medizin, Bern \\ ${ }^{2}$ Caresyntax, Berlin
}

\section{Background:}

Recent surgical literature shows that the level of surgical skill is associated with clinical outcomes. However, the assessment of surgical skills is time-consuming and often lacks objectivity. Therefore, we aim to achieve rater-independent objectivity in surgical skill assessment by using machine-learning algorithms.

\section{Materials and methods:}

Video recordings of laparoscopic cholecystectomies were segmented into procedural steps and annotated for specific surgical gestures like electric hook and clipping movements. The skill level of the surgical gestures were rated by three board certified surgeons using a Likert scale ranging from 1 (minimum) to 5 (maximum). Inter-rater reliability of surgical skill rating was assessed using a one-way random average-measure intraclass correlation coefficient (ICC).

A pretrained 3D residual convolutional neural network, which is a machine-learning model with spatiotemporal features, was trained on the curated dataset. Threefold cross-validation was performed to calculate the accuracy of the machine-learning algorithm in predicting surgical skill level. Accuracy was calculated by taking the ratio of correct predictions on each of the three folds, separately, and then averaged into a final score and reported.

\section{Results:}

144 electric hook and 524 clipping movements were annotated in 112 laparoscopic cholecystectomy videos. The mean \pm standard deviation surgical skill rating for electric hook movement was $3.52 \pm 1.93$ and for clipping $3.67 \pm 1.23$, respectively. Average measure ICC of surgical skill rating was $0.92(95 \%$ $\mathrm{Cl} 0.88-0.94)$. Our model predicted surgical skill level with an average accuracy of $82 \%$ and a mean absolute error of 0.28 .

\section{Conclusion:}

Expert rating of surgical skills shows an excellent inter-rater reliability. Machine-learning algorithms can be trained to predict surgical skill level from analysis of laparoscopic videos with a good accuracy. 
Machine learning variable selection determines significant predictors for surgical complications after upper $\mathrm{GI}$ resections

(Abstract ID: 674)

\author{
J.-O. Jung ${ }^{1}$, A. Knabe ${ }^{1}$, H. Nienhüser ${ }^{1}$, L. Peters ${ }^{1}$, B. P. Müller-Stich ${ }^{1}$, M. Büchler ${ }^{1}$, T. Schmidt ${ }^{1}$ \\ ${ }^{1}$ Universitätsklinikum Heidelberg
}

\title{
Background:
}

Postoperative complications are dreaded adverse events after oncologic resections of the esophagus and stomach. In Western countries, the adequate prediction of complications after upper GI surgery will become more relevant due to increasing numbers of esophageal and gastric carcinomas. Machine learning techniques can help determine significant associations based on complex and vast data. Retrospective machine learning studies suggest that artificial intelligence may be promising in case of multiple potential factors with unclear relations to each other. The aim of this study was to apply a random forest algorithm for variable selection to optimize prediction of surgical complications after upper Gl surgery.

\section{Materials and methods:}

In a prospectively maintained database, data of 1165 patients with gastric or esophageal cancer was collected who underwent oncologic resection between 2002 and 2019 at Heidelberg University Hospital, Department of General Surgery. We collected data about preoperative features, in-hospital course, laboratory parameters and eventually intraoperative or postoperative events. Out of a total of 251 variables, we performed variable selection via Boruta machine learning algorithm. Ordinal variables were dichotomized and we used median imputation for missing values. In multivariate analyses, we performed binary logistic regression on STATA SE 15.0 for Mac to determine relevant predictors out of the variables detected by the machine learning algorithm.

\section{Results:}

We were able to create models for a total of 43 variables classified as clinical complications. Figure 1 visualizes the Boruta machine learning algorithm for variable selection on the basis of anastomotic leakage as one of the 43 analyzed adverse events.

Multivariate analysis revealed significant predictors for abdominal abscess which were serum amylase on the day of operation $(p=0.044)$, on postoperative day one (POD 1, $p<0.001)$, serum lipase on POD $1(p=0.039)$, serum potassium on POD $1(p=0.017)$, leukocyte count on POD $2(p=0.006)$ and intraoperative extension $(p=0.002)$. Based on the model, a specificity of $99.53 \%$ and a negative predictive value of $93.33 \%$ could be reached.

For anastomotic leakage, significant predictors were serum C-reactive protein on POD $2(p<0.001)$, leukocyte count on POD $2(p=0.045)$, single or two cavity surgery $(p=0.036)$, gastric sleeve reconstruction $(p=0.045)$ and anastomosis in the lower mediastinum $(p=0.004)$. The specificity was calculated to be $98.78 \%$ with $86.29 \%$ of the cases correctly classified.

For pancreatic fistula, Leukocyte count $(p=0.048)$ and serum creatinine $(p=0.014)$ on the day of operation as well as serum amylase $(p=0.019)$ and creatinine on POD $1(p=0.014)$ were significant predictors primarily identified by the algorithm. Specificity was $99.82 \%$ and $98.32 \%$ of all patients could be correctly classified by the model.

These and further results such as mortality within and in-hospital mortality are summarized in Table 1. 
Table:

\begin{tabular}{|c|c|c|c|c|}
\hline Outcome variable & Predictor variables & $\begin{array}{l}\text { Odds Ratio (95\%- } \\
\mathrm{Cl})\end{array}$ & $p$-value & correctly classified \\
\hline abdominal abscess & $\begin{array}{l}\text { amylase POD } 0 \\
\text { amylase POD } 1 \\
\text { lipase POD } 1 \\
\text { potassium POD } 1 \\
\text { leukocytes POD } 2 \\
\text { surgical extension }\end{array}$ & $\begin{array}{l}0.987(0.975- \\
0.999) \\
1.014(1.006- \\
1.021) \\
0.991(0.983- \\
0.999) \\
2.215(1.151- \\
4.269) \\
1.106(1.030- \\
1.189) \\
3.091(1.511- \\
6.325)\end{array}$ & $\begin{array}{l}p=0.044 \\
p<0.001 \\
p=0.039 \\
p=0.017 \\
p=0.006 \\
p=0.002\end{array}$ & $92.96 \%$ \\
\hline $\begin{array}{l}\text { anastomotic } \\
\text { leakage }\end{array}$ & $\begin{array}{l}\text { CRP POD } 2 \\
\text { leukocytes POD } 2 \\
1 \text { or } 2 \text { cavity } \\
\text { gastric conduit } \\
\text { lower mediastinum }\end{array}$ & $\begin{array}{l}1.019(1.007- \\
1.015) \\
1.046(1.001- \\
1.094) \\
4.680(1.106- \\
19.797) \\
5.173(1.041- \\
25.706) \\
2.204(1.290- \\
3.767)\end{array}$ & $\begin{array}{l}p<0.001 \\
p=0.045 \\
p=0.036 \\
p=0.045 \\
p=0.004\end{array}$ & $86.29 \%$ \\
\hline pancreatic fistula & $\begin{array}{l}\text { amylase POD } 1 \\
\text { creatinine POD } 1 \\
\text { creatinine POD } 0 \\
\text { leukocytes POD } 0\end{array}$ & $\begin{array}{l}1.010(1.002- \\
1.018) \\
25.332(1.918- \\
334.552) \\
0.031(0.002- \\
0.493) \\
1.067(1.001- \\
1.137)\end{array}$ & $\begin{array}{l}p=0.019 \\
p=0.014 \\
p=0.014 \\
p=0.048\end{array}$ & $98.32 \%$ \\
\hline 30d mortality & $\begin{array}{l}\text { blood transfusion } \\
\text { creatinine POD } 2\end{array}$ & $\begin{array}{l}1.750(1.114- \\
2.750) \\
4.157(1.421- \\
12.158)\end{array}$ & $\begin{array}{l}p=0.015 \\
p=0.009\end{array}$ & $98.10 \%$ \\
\hline in-hospital mortality & $\begin{array}{l}\text { creatinine POD } 2 \\
\text { leukocytes POD } 2 \\
\text { R0 / RX status mid } \\
\text { esophageal CA }\end{array}$ & \begin{tabular}{|l|}
$4.034(1.814-$ \\
$8.970) 1.121(1.053$ \\
$-1.194) 2.180$ \\
$(1.341-3.542)$ \\
$6.211(2.514-$ \\
$15.345)$
\end{tabular} & $\begin{array}{l}p=0.001 p<0.001 \\
p=0.002 p<0.001\end{array}$ & $96.21 \%$ \\
\hline
\end{tabular}

Table 1

\section{Conclusion:}

The clinical relevance of the above-mentioned tests needs to be clarified in prospective analyses. In the future, the presented data could enable accurate screening tests based on machine learning. By demonstrating the significance of the predictor variables which were identified by Boruta, we could show that the algorithm was able to intelligently select predictors and we thus deliver a proof of concept concerning the feasibility and validity of big data analysis for surgical complications. 


\section{Picture:}

Boruta random forest algorithm - Anastomotic leakage

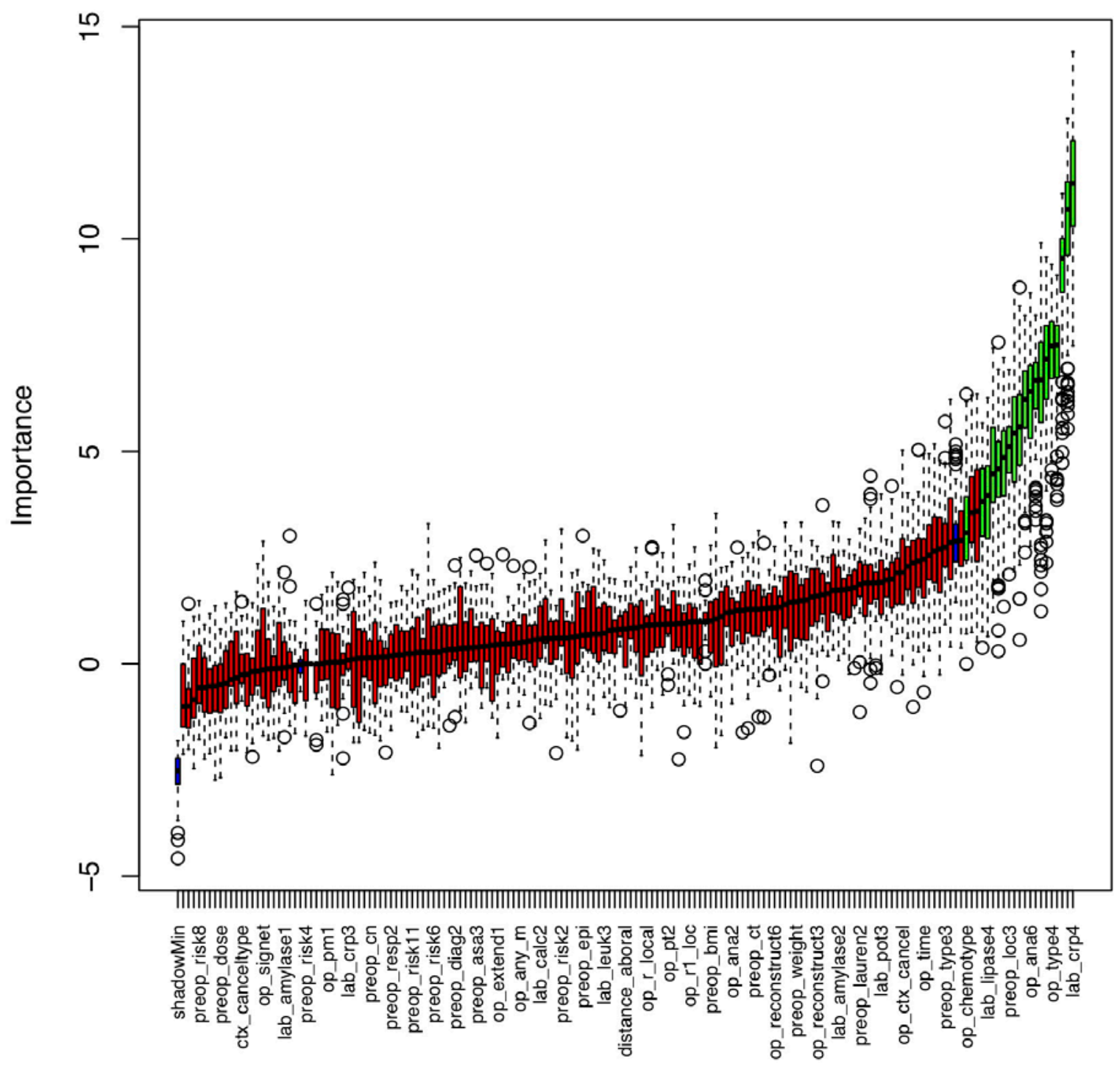

Figure 1 


\section{Hyperspectral imaging (HSI) for tumor cell identification and classification in Barrett's carcinoma patients}

(Abstract ID: 802)

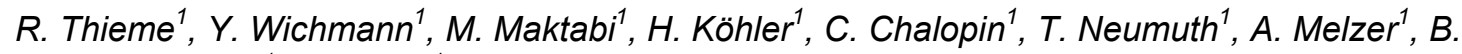
Jansen-Winkeln ${ }^{1}$, I. Gockel ${ }^{1}$

${ }^{1}$ Universitätsklinikum Leipzig

\section{Background:}

Hyperspectral imaging (HSI) technology combines imaging with spectroscopy and can be used to identify and classify malignant and non-malignant cells from histologically specimens.

\section{Materials and methods:}

Esophageal adenocarcinoma and squamous epithelium were recorded by HSI between the visual and near-infrared light $(500-1000 \mathrm{~nm})$. The specimens were fixed in $4 \%$ formaldehyde after resection and slices were conducted $(3 \mu \mathrm{m})$, which were stained with haematoxylin and eosin (HE). K-nearest neighbours (k-NN), a non-parametric supervised classification learning algorithm, logistic regression and supported vector machine (linear) were used for discrimination.

\section{Results:}

We were able to record esophageal adenocarcinoma cells by HSI. Differences in the absorbance of squamous epithelium and esophageal adenocarcinoma cells were determined at the wave lengths of $530 \mathrm{~nm}$ and $590 \mathrm{~nm}$, which represent eosin's and hematoxilin's maximal absorption, respectively. The intra group variances were quite low, for the squamous epithelium as for the esophageal adenocarcinoma cells. For squamous epithelium 74,000 and for esophageal adenocarcinoma cells 333,275 spectra were measured. K-nearest neighbours (k-NN), logistic regression and supported vector machine revealed different sensitivities and specificities for tumor cell classification.

\section{Conclusion:}

Squamous epithelium and esophageal adenocarcinoma cells show specific spectral alterations due to their staining with HE, when measured by HSI. However, the training algorithms need to be validated to foster decision-making support in histopathological diagnosis. 


\section{Predictive factors of early outcome after palliative surgery for colorectal carcinoma}

(Abstract ID: 413)

R. Konopke ${ }^{1}$, J. Schubert ${ }^{1}$, O. Stoeltzing ${ }^{1}$, S. Kersting ${ }^{2}$

${ }^{1}$ Elblandklinikum Riesa

${ }^{2}$ Universitätsklinikum Erlangen

\section{Background:}

A significant number of patients with colorectal cancer present with various conditions requiring surgery in an oncologically palliative setting. This study was performed to identify risk factors for early outcome after the operation in order to identify factors to aid the decision on management.

\section{Materials and methods:}

We performed a retrospective chart review of 142 patients who underwent palliative surgery due to local advanced, complicated or metastatic colorectal carcinoma between January 2010 and April 2018 at the "Elbland" Medical Center Riesa. Logistic regression analysis of 43 factors was performed to identify independent predictors for the length of hospital stay, complications and mortality.

\section{Results:}

Operations included resections with primary anastomosis $(n=31 ; 21.8 \%)$ or discontinuous resections with colostomy $(n=38 ; 26,8 \%)$, internal bypasses $(n=27 ; 19.0 \%)$ or stoma formation only $(n=46$; $32.4 \%)$.

The median length of hospital stay was twelve days ( 2 - 53 days), in-hospital morbidity and mortality rates were $50.0 \%$ and $18.3 \%$, respectively.

Preoperative anticoagulation (HR: 7.000; $\mathrm{p}=0.011$ ) and renal failure (HR: $1.377, p=0.038$ ) were independent risk factors for a prolonged hospital stay in multivariate analysis.

Independent risk factors of in-hospital morbidity were age (HR: 1.468, $p=0.046)$ and different comorbidities of the patients [obesity (HR: 1.786, $p=0.036$ ), renal failure (HR: 1.606, $p=0.040$ ), diabetes (HR: 1.596, $p=0.032)$, alcohol abuse (HR: 1.306, $p=0.023)$ as well as lung metastases (HR: $1.578, p=0.041)$.

Emergency surgery (HR: 10.216, $p=0.019$ ), ileus (HR: 9.180, $p=0.006)$, alcohol abuse (HR: 6.900, $p=0.003$ ), nicotine abuse (HR: 3.990, $p=0.032$ ) and ascites (HR: $2.100, p=0.034$ ) were found to be multivariately significant parameters of in-hospital mortality.

\section{Conclusion:}

Palliatively treated patients with colorectal cancer undergoing surgery show high rates of morbidity, mortality and a prolonged hospital stay after surgery. Predictive factors for the early outcome are comparable to patients in curable situations. An adequate selection of patients before palliative operation should lead to a better outcome. Especially in older patients with emergency surgery, history of drink and tobacco abuse and/or ascites, the safest possible strategy should be employed. 


\section{Divergent lung function in elective upper limb surgical procedures}

(Abstract ID: 174)

F. Lingad' ${ }^{1}$ M. Lange ${ }^{2}$, S. Böhle ${ }^{2}$, G. Matziolis ${ }^{2}$, S. F. Hertling ${ }^{2}$

1 , Jena

${ }^{2}$ Waldkliniken Eisenberg

\section{Background:}

Regional anesthesia procedures are often used to ease perioperative pain management in upper limb surgery. This can lead to serious complications causing cardio-pulmonary impairment. The recent development of the health care system leads to more ambulant operations with regional anesthetic procedures, even in senior citizens. The aim of this study is to investigate the impact of plexus anesthesia (PA) on cardio-pulmonary performance unrelated to complications. Furthermore we evaluated to what extent the postoperative outcome and the patient's age are important for perioperative risk management.

\section{Materials and methods:}

90 patients were examined. Patients in the control group (CG) received elective orthopedic surgery (n $=30$ ). The study group (SG) includes patients undergoing elective procedures on the upper extremity. Surgery procedures with (SG I, $n=30$ ) and without PA (SG II, n=30) are assigned in two separate groups. Target parameters were, in addition to the vital parameters, the FEV-1 and the PEF, pCO2, $\mathrm{pO} 2, \mathrm{pH}, \mathrm{BE}, \mathrm{HCO}-$. The measured values were determined by means of capillary blood gas analysis and spirometry measurement. These were performed simultaneously pre / intra / postoperatively after specified times.

\section{Results:}

The lung function was impaired in all three groups postoperatively. The pulmonary functions of the SG are worse postoperatively than those of the CG. Within the SG, the SG II shows a better cardiopulmonary performance intraoperatively and postoperatively than the SG I. Patients of the SG I, who were older than 60 years, had a significantly higher impairment of lung function than the control group.

\section{Conclusion:}

Operative interventions aggravate cardio-pulmonary performance. The PA seems to have an impact on the cardio-pulmonary efficiency and has a negative influence on the lung function. Elderly patients are more affected. This should be considered as part of the perioperative management, especially from a surgical point of view. 
Do we know how good we are? An approach to the systematic documentation of adverse events in a tertiary referral centre for plastic surgery

(Abstract ID: 518)

\author{
S. Leuschner ${ }^{1}$, C. C. Corterier ${ }^{1}$, F. Siemers ${ }^{1}$ \\ ${ }^{1}$ BG Klinikum Bergmannstrost Halle
}

\title{
Background:
}

In surgery, adverse events are rarely systematically documented. Previous retrospective research has shown that adverse events occur in $10-45 \%$ of patients in hospital. Up to half of these are deemed to be preventable.

Since complications are relatively common, it seems plausible that recurring patterns can be identified, if adverse events are systematically documented. The hospital environment is a complex system and adverse events are - at least in part - a product of the system. It has been proposed that adverse events are rarely due to individual error alone but are more likely to be the result of the processes and resources of the system.

Systematically identifying adverse events could enable us to identify recurring patterns so that the underlying structural reasons for such events can be addressed. The results of such a structural change can then be assessed by continued monitoring of adverse events.

In clinical practice, adverse events are sometimes discussed at morbidity and mortality meetings. The cases presented in these meetings are often selected for their uniqueness or severity of the adverse event. In our experience, these meetings rarely result in meaningful change. Even if changes are implemented, their consequences cannot be monitored. In addition, the vast majority of adverse events cannot be discussed in such a setting.

We describe the successful implementation of a system to identify and document adverse events in a tertiary referral centre for plastic surgery and discuss the challenges and pitfalls encountered in its implementation.

Systematic documentation of adverse events is possible in a tertiary referral centre for plastic surgery. 


\section{Report of a quality improvement program for reducing postoperative complications by using a surgical risk calculator in a cohort of general surgery patients}

(Abstract ID: 814)

E. Müller ${ }^{1}$

${ }^{1}$ Asklepios Klinik Langen

\section{Background:}

Improving perioperative care and reducing postoperative complications is important at a time when the number of surgeries and their associated costs are increasing each year. Various quality improvement programs as the WHO Surgery Safety checklist address the problem. Also, the surgeons' perception of potential risk vary wildely. The ACS NSQIP Surgical Risk Calculator ${ }^{\circledR}$ as a computer decision support system was designed to debias surgical strategy for better risk assessment and treatment decisions in a shared-decision-making process.

This study determines whether a surgical risk calculator can reduce postoperative complications in elective surgery via raising the awareness of surgeons for postoperative complications in a preoperative briefing.

\section{Materials and methods:}

The risk calculator was employed retrospectively in the period 2012/2013 in one group ('Retro'), and prospectively in the other group ('Prosp') in the period 2014/2015. Each group consisted of 832 patients. Patients were selected by propensity score matching. To determine whether the preoperative usage of the SRC could reduce postoperative complications, actual postoperative complications of the two groups were compared. The complications were classified by the ACS NSQIP Surgical Risk Calculator ${ }^{\circledR}$ complication categories and the Clavien-Dindo complication classification system.

In addition sensitivity and specificity were calcultated to determine if there was a destinction between the risk calculator's predictions ('Prosp'-group) and the doctor's perception ('Retro'-group) for possible postoperative complications. Positive and negative predictive values were calculated for both groups to find out how high the probability of the result accuracy was and if there were any differences between the two groups outcome reliability.

\section{Results:}

Comparing ACS NSQIP Surgical Risk Calculator ${ }^{\circledR}$ "Serious Complication" and "Any Complication" no significant increase in the 'Prosp' group was apparent (Serious Complication: $6.6 \%$ vs. $8.5 \%, p=$ 0.164; Any Complication: $8.5 \%$ vs. $9.7 \%, p=0.444$ ). Complications in the 'Retro' vs. 'Prosp' groups, according to the Clavien-Dindo I-V classification, were: I - $17.9 \%$ vs. $20.0 \%$; II - $7.4 \%$ vs. $6.0 \%$; III $2.5 \%$ vs. $2.6 \%$; IV $-1.0 \%$ vs. $1.9 \%$; V $-1.0 \%$ vs. $1.3 \%, p=0.403$, respectively. Results showed a medium specificity $(74.5 \%$ vs $75.0 \%, p=0.478)$ and poor sensitivity $(40.6 \%$ vs. $39.7 \%, p=0.806)$. The positive predictive value was low $(4.2 \%$ vs. $4.0 \%, p=0.790)$. The negative predictive value showed good results $(98.1 \%$ vs. $97.4 \%, p=0.016)$.

\section{Conclusion:}

The study did not show that using a surgical risk calculator could reduce postoperative complications due to increased risk awareness of the surgeons. This could be due to the poor sensitivity and medium specificity of the risk calculator in a different health care system than it was originally developed. Also, since the observed complication rate of this study was low, it is possible that 
reductions of complications are not easily achieved in an environment of already highly implemented risk management tools. 


\section{Possibilities and limits of the Da Vinci robotic system in revisional bariatric surgery}

(Abstract ID: 161)

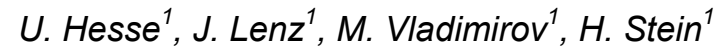

${ }^{1}$ Klinikum Nürnberg Paracelsus Medizinische Privatuniversität

\section{Background:}

The arrival of robotic assisted surgery in the treatment of morbidly obese patients has enlarged the armamentarium for surgeons involved in bariatric surgery.

This in particular is of great advantage not only in primary cases but also in patients undergoing revisional procedures following preceeding upper GI surgery.

Aim: In the following the experience with revisional surgery using the Da Vinci robotic system will be reported and compared to conventional laparoscopic treatment and the literature.

\section{Materials and methods:}

In a 30 months period a total of 134 minimally invasive bariatric procedures (42 robotic assisted, 92 laparoscopic) were performed. 47 patients received a gastric bypass, 71 a gastric sleeve and in 16 patients a band was removed or adhesions were resected without an alternative procedure. Out of the 47 GBP procedures $30(63 \%)$ were performed robotic. Out of these $15(50 \%)$ had previous operations 1 hiatal mesh repair, 1 open Mason operation, 6 gastric band, 6 gastric sleeve and 1 gastric sleeve with fundoplication. The Da Vinci Xi was used for the surgery.

The conversion rate, operating time, surgical complications, postoperative stay in days were analyzed in the patients undergoing robotic Roux-Y gastric bypass as redo procedure (Group 1) and compared to patients undergoing robotic Roux- $Y$ gastric bypass as primary operation (Group 2).

\section{Results:}

In $3 / 15(20 \%)$ of all patients undergoing revisional robotic assisted surgery (group 1 ) the procedure had to be converted to open surgery due to intraabdominal abnormalities ( 1 oversized left liver lobe, 1 extreme adhesions and 1 short mesentery). $1(7 \%)$ patient had to be reoperated for insufficiency of the biliodigestive anastomosis.

In the patients without previous surgery undergoing robotic Gastric bypass ( $n=15$, group 2$) 1(7 \%)$ patient had to be converted to laparoscopy for adhesions. However $3(20 \%)$ patients had to be reoperated for bleeding 1, biliodigestive anastomosis 1 and insufficiency of the gastroenterostomy 1 , 2and 3 days following the primary operation. The average operating time was 194,5 min (142-228) (group 1) vs 290,2 min (154-480) (group 2).

There was no mortality in both groups and reoperated patients remained in the hospital 12,3 days as compared to 3,5 days in patients without complications.

The overall complication rate including laparoscopic and robotic cases was 6/134 (5,9\%) 4 bleedings and 2 suture insufficiences. One patient $(0.7 \%)$ died 22 days following sleeve gastrectomy because of rhabdomyolysis.

\section{Conclusion:}

Conclusion: The data suggest that robotic revisional surgery can be performed even in complicated cases however conversion to open surgery may be indicated when anatomical abnormalities are present. 


\title{
Features of haptic feedback in surgery - comparison of various surgical manipulators
}

(Abstract ID: 966)

\author{
J. Rolinger ${ }^{1}$, W. Kunert ${ }^{1}$, J. Bilz², K. Jansen ${ }^{1}$, J. Miller ${ }^{1}$, J. Johannink ${ }^{1}$, H. Schlaak ${ }^{2}$, A. Kirschniak ${ }^{1}$ \\ ${ }^{1}$ Universitätsklinikum Tübingen \\ ${ }^{2}$ Technische Universität Darmstadt
}

\section{Background:}

The value of haptic feedback in minimally invasive and robot-assisted surgery remains controversial. The further development of robotic systems increasingly provides solutions that can simulate a tactile perception even in teleoperating setups. To what extent this additional information is relevant for carrying out a specific surgical procedure is currently difficult to answer. The aim of the present study was to investigate the haptic perception properties of various surgical manipulators with regard to the assessment of different forces. The investigation was carried out using the FLEXMIN teleoperated surgical robot (DFG project KI1645 / 1-3).

\section{Materials and methods:}

The randomized cross-over study compares 5 surgical manipulators (fingertips, conventional forceps, laparoscopic forceps, robotic forceps with or without haptic feedback) with respect to the ability to discriminate certain forces (hardness estimation and tensile force comparison). The study group included 25 medical students and 10 surgical experts.

\section{Results:}

Concerning hardness estimation in the novice group, the mean number of correct answers out of 9 possible was 6 in manual mode $(p<0.05), 5$ in conventional mode $(p<0.05), 3$ in laparoscopic mode, 4 in robotic mode without haptic feedback and 3 in robotic mode with haptic feedback (each without significant difference). Concerning tensile force comparison in the novice group, a difference of $>=5$ $\mathrm{cN}$ in manual mode, of $>=10 \mathrm{cN}$ in conventional mode, of $>=50 \mathrm{cN}$ in laparoscopic mode, of $>=30 \mathrm{cN}$ in robotic mode with haptic feedback and of $>=50 \mathrm{cN}$ in robotic mode without haptic feedback could be assigned correctly. In the expert group, only robotic mode with and without haptic feedback were compared. In the hardness estimation setup, the haptic feedback had no significant advantage. Regarding the tensile force comparison, a difference of $>=30 \mathrm{cN}$ in robotic mode with haptic feedback could be assigned correctly, whereas in robotic mode without haptic feedback no difference could be assigned correctly.

\section{Conclusion:}

The conventional surgical instruments were consistently superior to the minimally invasive and robotic manipulators in the force estimation comparison. The use of haptic feedback significantly improved the results of the surgical robot in the novice group regarding the estimation of tensile forces. Further studies are needed to investigate the impact of haptic feedback on efficiency and safety in minimally invasive surgery. 


\section{Priority Setting Partnership (PSP) Pancreatic Cancer - the "TOP 10" research questions on therapy of pancreatic cancer}

(Abstract ID: 354)

R. Klotz ${ }^{1}$, C. Doerr-Harim ${ }^{1}$, C. Tjaden ${ }^{1}$, A. L. Mihaljevic ${ }^{1}$

${ }^{1}$ Universitätsklinikum Heidelberg

\section{Background:}

Pancreatic cancer is the fourth leading cause of cancer deaths in Germany. Research projects on cancer are currently initiated by industry or scientists, usually without involving relevant stakeholders (treating physicians, patients, family members, caregivers, nurses, etc.). However, there is a mismatch between available research evidence and the research preferences of personally affected people, i.e. patients and caregivers. Consequently, involving patients needs to be an upcoming topic in clinical research and health-care politics.

\section{Materials and methods:}

Priority Setting Partnerships (PSPs) aim to involve patients, caregivers, doctors and other relevant stakeholders as equal partners to find the most urgent unanswered research questions for a certain disease. This is achieved by a transparent 7-step process including survey, literature reviews and prioritisations. The PSP pancreatic cancer was initiated in Heidelberg in cooperation with the British James Lind Alliance (JLA), a non-profit making initiative, which was established in 2004 and completed its first PSP in 2007. The aim is to identify and prioritise the ten most urgent open research questions / uncertainties on treatment of the disease. These "TOP 10" questions will be published to stimulate and steer future patient-orientated research.

\section{Results:}

In the beginning of 2017 a steering group was established in Heidelberg involving representatives of all relevant stakeholders in order to design and to decide equally about all relevant aspects. The first nation-wide survey of the PSP pancreatic cancer was carried out from August to November 2017 and revealed 566 questions submitted by 140 participants regarding questions about the therapy of the disease. After removal of duplicates and out of scope questions, uncertainties were checked against current evidence. The remaining open research questions / uncertainties were included in the second nation-wide survey for interim prioritization accomplished from June to September 2019 by 211 participants (107 patients/relatives and 104 health care professionals). The final face-to-face consensus conference will take place in December 2019. The thereby achieved "TOP 10" list of the PSP pancreatic cancer will be presented at congresses as well as at patient representative meetings and published in medical journals and lay magazines.

\section{Conclusion:}

The identified questions warrant realised patient involvement and will initiate patient-relevant research and research funding, thus improving the care of those most affected by pancreatic cancer. The presented PSP establishes the transparent, validated JLA method for the first time in Germany and is the first PSP on the subject of pancreatic cancer world-wide. Valuable experience from the implementation of this project will help future German PSPs on other diseases and further projects with the aim to strengthen patient participation. 


\section{Evidence Map of Pancreatic Surgery - Living Systematic Review and Meta-Analysis}

(Abstract ID: 433)

P. Probst ${ }^{1}$, F. Hüttner ${ }^{1}$, Ö. Meydan ${ }^{1}$, E. Kalkum ${ }^{1}$, A. L. Mihaljevic ${ }^{1}$, T. Hackert ${ }^{1}$, M. Büchler ${ }^{1}$, M. Diener ${ }^{1}$

${ }^{1}$ University of Heidelberg

\section{Background:}

Pancreatic surgery is a large and complex field of research. Several evidence gaps exist for specific diseases or surgical procedures. An overview on existing knowledge is needed to plan and prioritise future research. The aim of this project is to create a systematic and living evidence map of pancreatic surgery.

\section{Materials and methods:}

PubMed, CENTRAL and Web of Science were searched systematically for all randomised controlled trials (RCT) and systematic reviews (SR) on pancreatic surgery. Outcomes and quality of every existing RCT were extracted. SR were used to identify lack of high-quality trials. A web-based evidence map in the format of a mind map was created including meta-analyses of pancreas-surgery specific outcomes for every research topic.

\section{Results:}

From over $30^{\prime} 000$ articles 246 RCT on $26^{\prime} 154$ patients and 220 SR were included and grouped in over 50 different research topics. Most trials were conducted in Europe (46\%), followed by Asia (35\%) and North America (14\%). As an example, the pooled rate of postoperative pancreatic fistula in 9555 partial pancreatoduodenectomy patients was $15 \%(99 \%-\mathrm{Cl} 12 \%-17 \%)$. The overall quality of the included RCT was moderate. A lack of evidence exists for operations other than pancreatoduodenectomy and for specific diseases such as neuroendocrine neoplasms or intraductal papillary mucinous neoplasms.

\section{Conclusion:}

The evidence map of pancreatic surgery gives a systematic and comprehensive overview of the available literature. The pooled estimates of outcomes provide prospectively assessed benchmarks. The intuitive display of the existing literature uncovers evidence gaps and serves as an objective basis for decisions about future research priorities. The web-based evidence map will be accessible in spring 2020 (www.evidencemap.surgery). 


\section{Lean Evidence Management - Applicability and Feasibility of an Industrial Management Principle in Clinical Research}

(Abstract ID: 434)

M. Diener ${ }^{1}$, F. Hüttner ${ }^{1}$, P. Knebel ${ }^{1}$, A. L. Mihaljevic ${ }^{1}$, P. Probst ${ }^{1}$

${ }^{1}$ University of Heidelberg

\section{Background:}

Clinical research is faced with the identical economic pressure as the whole medical system. The current organization of clinical research lacks of centralized prioritization of urgent and relevant scientific research questions. As a result, evidence is produced today by chance or due to the pressure of so-called megatrends. Besides impaired process quality of the whole evidence production, process shortness of financial and personnel resources are wasted for scientific projects of inferior originality and relevance (research waste).

\section{Materials and methods:}

Aim of this empirical study was the evaluation of the applicability of the industrial management principles of Lean Management in clinical research. Literature searches were done in order to assess the current body of evidence of research management with a special focus on the terms prioritization and waste reduction in clinical research. The principles of the management philosophy of lean management were applied to the process of evidence production in clinical research.

\section{Results:}

This study showed that the keywords production, quality, competitiveness, innovation and economics are evenly important for industrial and evidence production in research. The system of Lean Evidence Management was created by the interdependent incorporation of Evidence Mapping, Prioritization, Scientific Communication and Knowledge Transfer. The central element of evidence mapping was framed and visualized exemplarily for pancreatic surgery (www.evidencemap.surgery). All analyzes showed high applicability of the Lean Management principles in clinical research.

\section{Conclusion:}

Besides feasibility, effectiveness and increase of output and quality is most likely. With Lean Evidence Management a new term and concept was created, which is currently not available in the literature. 


\title{
CT-guided thoracic sympathicolysis versus video-assisted thoracoscopic sympathectomy in the therapeutic concept of severe primary palmar hyperhidrosis
}

(Abstract ID: 30)

\author{
J. R. Andresen ${ }^{1}$, F. Scheer ${ }^{1}$, E. Schlöricke ${ }^{1}$, A. Sallakhi ${ }^{1}$, M. O. Liedke ${ }^{1}$, R. Andresen ${ }^{1}$ \\ ${ }^{1}$ Sigmund Freud University, Vienna
}

\section{Background:}

The clinical picture of primary palmar hyperhidrosis is characterised by excessive sweating beyond physiological levels, which can consecutively lead to considerable distress.

The objective of the present study was to compare the methods of CT-guided thoracic sympathicolysis (CTSy) and video-assisted thoracoscopic sympathectomy (VATS) with regard to their feasibility, the occurrence of minor and major complications and the clinical outcome.

\section{Materials and methods:}

Retrospectively, 78 patients (mean age $36.4+/-15$ years) who had been treated using CTSy and 74 patients (mean age $34.2+/-11$ years) who had been treated using VATS were included in the study. The indication for treatment was primary focal palmar hyperhidrosis after exhaustion of all conservative treatment options and a continued high level of suffering.

For both groups, the interventions were performed exclusively unilaterally, the contralateral side being treated after approx. 6 weeks.

CTSy was performed after establishing the entry plane at the level of the intervertebral space Th $2 / 3$ via a dorsolateral approach using a 22-G coaxial needle. On average $5(2-8) \mathrm{ml}$ of a sympathicolytic mixture consisting of $96 \%$ alcohol, $0.5 \%$ Carbostesin and $0.9 \% \mathrm{NaCl}$ solution with added amounts of contrast medium were instilled.

VATS was performed under intubation anaesthesia with a double lumen tube for single-lung ventilation, whereby the respective side of the lungs was collapsed. After insertion of the instruments via a minithoracotomy, the parietal pleura was dissected and the sympathetic trunk severed below Th 2.

All patients evaluated their sense of discomfort within the context of a quality control before the start of treatment, two days postinterventionally / postoperatively, and six and twelve months after treatment, on the basis of a Dermatology Quality of Life Index (DLQI) as well as the side effects that occurred. As statistical test procedures, the Wilcoxon rank-sum test was applied to test the differences in DLQI values over the course and the Mann-Whitney $U$ test to compare the different methods.

\section{Results:}

Both methods were technically fully feasible in all patients. Both treatments led to a marked reduction in the preinterventional sense of discomfort two days postinterventionally / postoperatively as well as six and twelve months after treatment $(p<0.001)$, whereby mild recurrent sweating occurred over the further course, which was significantly higher in the patients who had undergone CTSy. Short-term miosis and ptosis was found in 8 of $78(10.3 \%)$ patients after CTSy and in 6 of $74(8.1 \%)$ patients after VATS. As the most common side effect, transient compensatory sweating, predominantly on the trunk, was reported by 16 of $78(20.5 \%)$ patients after CTSy and 10 of $74(13.5 \%)$ patients after VATS. Pneumothoraces, caused by fistula formation after adhesiolysis, developed postoperatively in 6 of 74 
(9.1\%) cases, which were treated adequately by means of a drain already inserted intraoperatively. Temporary pain after thoracotomy was experienced by 12 of $74(16.2 \%)$ patients.

\section{Conclusion:}

For patients with severe palmar hyperhidrosis, CTSy and VATS represented a minimally invasive treatment option that provided a high and largely equivalent level of benefit. Recurrent sweating is slightly more common and more pronounced after CTSy than after VATS. However, a higher rate of major complications is seen in the case of VATS. 


\title{
25 years experience with transpedal lymphangiography in the management of postoperative therapy-refractory lymphatic leakage - final results with subgroup analyses
}

\author{
(Abstract ID: 159)

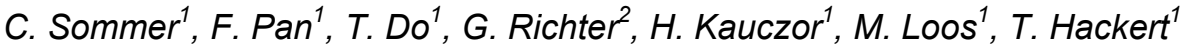 \\ ${ }^{1}$ University Hospital Heidelberg \\ ${ }^{2}$ Stuttgart Clinics
}

\section{Background:}

During the DCK (Deutscher ChirurgenKongress) 2019, the preliminary results of our 25 year experience on transpedal lymphangiography in the management of postoperative therapy-refractory lymphatic leakage were presented. In this update, the final results with subgroup analyses are summarized.

\section{Materials and methods:}

The final cohort consisted of 355 patients who underwent transpedal lymphangiography with intention to cure postoperative therapy-refractory lymphatic leakage in different locations. Study goals included patient demographics and technical results as well as complication and cure rates. Dedicated subgroups analyses were performed to outline predictors for cure.

\section{Results:}

Postoperative lymphatic leakage resulted from different in part highly complex surgical procedures in the neck, thorax, abdomen, pelvis and/or lower extremity. The daily drainage volume was $1124 \pm 1336 \mathrm{ml}$. Technical success rate of, amount of injected iodized oil used for, and major and minor complication rates of transpedal lymphangiography were $87.2 \%, 10.3 \pm 4.3 \mathrm{ml}$, and $0 \%$ and $0.3 \%$, respectively. The cure rate of transpedal lymphangiography was $42.6 \%$. Positive predictors for cure were radiological extravasation/pooling of iodized oil under fluoroscopy, radiography and especially CT (yes vs. no; $p=0.006$ ), type of lymphatic leakage (lymphatic fistula vs. lymphocele; $p=0.033$ ), and the daily drainage volume $(845 \pm 1067$ vs. $1316 \pm 1399 ; p=0.001)$.

\section{Conclusion:}

Transpedal lymphangiography is feasible, safe, and effective in the management of postoperative therapy-refractory lymphatic leakage. Under consideration of positive predictors for cure, different lymphatic second-line interventions should be scheduled prospectively, because according to our data lymphatic second-line interventions can increase dramatically the cure rate (e.g. CT-guided ethanol $95 \%$ sclerotherapy shows a cure rate of $76.9 \%$ after clinically ineffective transpedal lymphangiography). 


\section{Picture:}

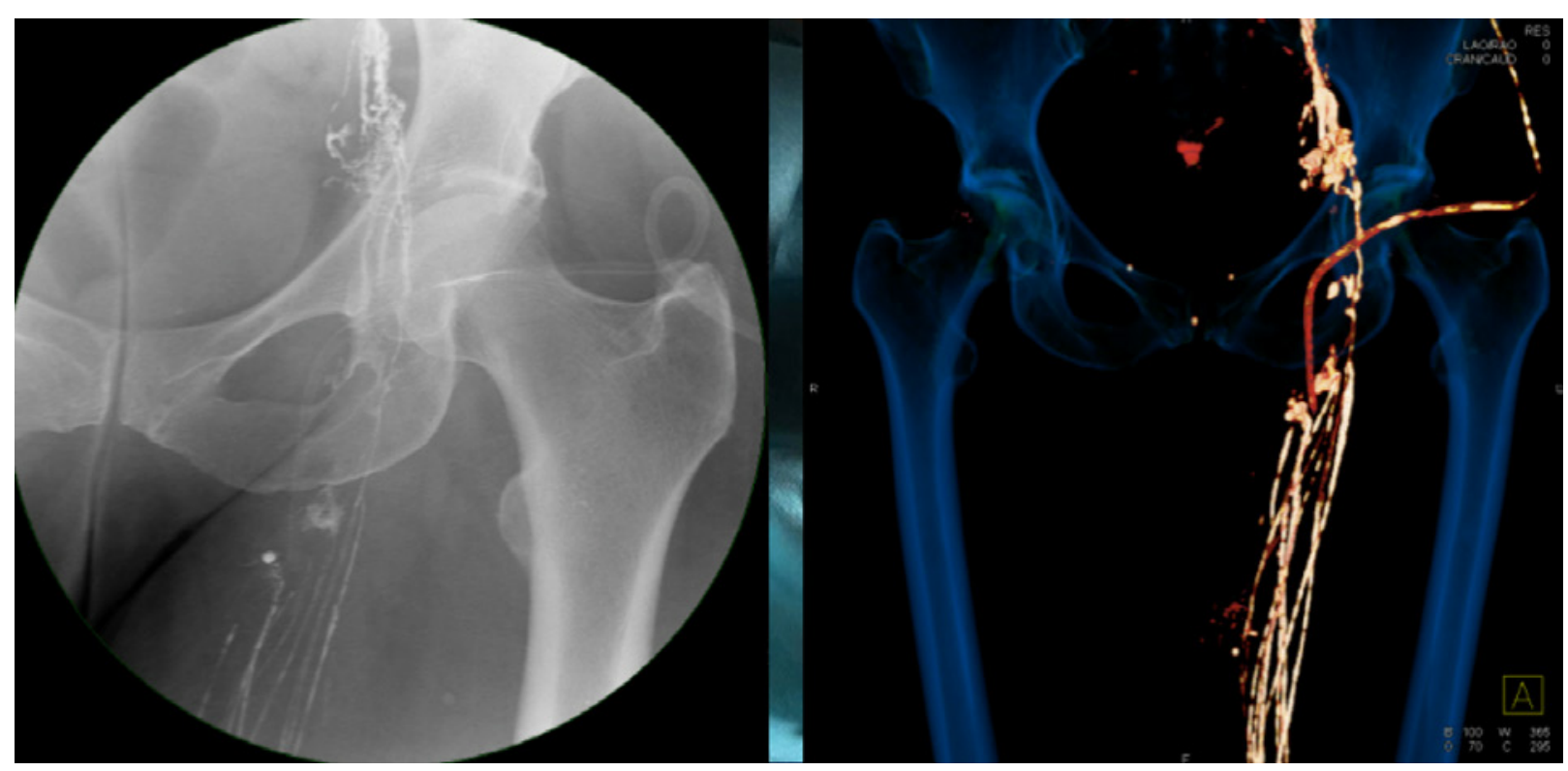

Therapeutic conventional (left) and CT (right) lymphangiography with iodized oil in a patient with postoperative lymphatic leakage after inguinal lymphadenectomy 


\section{Characterization of pain therapy aspects at a normal surgical ward of a tertiary center}

(Abstract ID: 540)

M. Brinkers ${ }^{1}$, M. Istel ${ }^{1}$, G. Pfau ${ }^{1}$, F. Meyer ${ }^{1}$

${ }^{1}$ Universitätsklinikum Magdeburg

\section{Background:}

To achieve best medical care by pain therapy has been seriously pursued in Germany for the last several years.

However, publications of the last decade may indicate that this challenging aim has not been achieved yet.

\section{Materials and methods:}

At a normal surgical ward of a university hospital (tertiary center), all consecutive patients were asked to fill out a standardised pain questionnaire at the admission to the hospital over a time period of 6 months. In addition, indication for surgical intervention, medication, pain score (according to VAS scale) and length of hospital stay were recorded. This systematic clinical prospective observational study (design) to reflect daily clinical practice and as contribution to quality assurance (and research on clinical care) was permitted by the institutional ethic committee

\section{Results:}

The most present pain score of the 269 patients enrolled in the study at admission was zero. The vast majority of subjects, in particular, 193 patients underwent surgical intervention. Their pain score could be significantly reduced from 1.9 at admission down to 1.2 at discharge ((mean each; $p=0.004)$. Interestingly, 71 patients showed a pain score of $>4$ at admission. During their hospital stay, the pain score could be significantly lowered from 6.3 in average to $1.8(p<0.001)$.

\section{Conclusion:}

At the investigated surgical ward, pain score after surgery could be effectively reduced, which contributed substantially to a higher level of surgical care in daily practice.

Registering pain as a routine procedure and subsequent or derived initiation of pain therapy on an adequate level are crucial in the majority of cases as part of daily clinical practice. 


\section{Pain consultation at a normal surgical ward of a tertiary center}

(Abstract ID: 541)

M. Brinkers ${ }^{1}$, M. Istel ${ }^{1}$, G. Pfau ${ }^{1}$, F. Meyer ${ }^{1}$

${ }^{1}$ Universitätsklinikum Magdeburg

\section{Background:}

As already reported from other medical disciplines, it has been proven that there is only a small percentage of patients who need consultation (e.g., in addition by psychiatry) for optimization of pain therapy.

\section{Materials and methods:}

At a university hospital normal surgical ward, all consecutive patients were asked to fill out a standardised pain-associated questionnaire at the time of their admission to the hospital over a time period of 6 months. In a regular follow-up examination, the effect of a former pain-therapeutic consultation was critically assessed and systematically evaluated.

This systematic clinical prospective observational study (design) to reflect daily clinical practice and as contribution to quality assurance (and research on clinical care) was permitted by the institutional ethic committee.

\section{Results:}

21 of 269 patients got a pain-therapeutic consultation $(7.8 \%)$.

In these patients, pain score could not be significantly reduced (VAS: 2.6 down to 1.6; $p=0.238$ ). Initial analyses revealed that these patients belong to a subgroup of patients (length of stay: 14.2 days versus the whole group: 7.2 days; percentage of cancer patients, $76.2 \%$ - in total: $35.3 \%$ ).

\section{Conclusion:}

The number of consulation is low, however, it is better than in psychiatric patients (consultation rate, 2.5 to $3.0 \%$ ). Obviously, the patients who achieved a pain consultation were not be treated sufficiently. This is in contrast to the previous results on the effect of pain-therapeutic consultations at our outpatient clinic ( $n=386$ ). It could be, therefore, a numerical problem, which needs to be taken into account. In addition, the criteria for requesting a consultation are still unclear. Clearly, the consultation procedure has to be optimized. 


\section{Annexin A1 expression capacity as a determinant for disease severity in Crohn's disease}

(Abstract ID: 883)

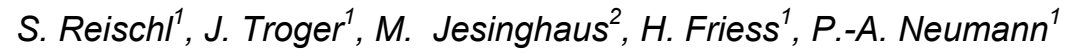

${ }^{1}$ Klinikum rechts der Isar, München

${ }^{2}$ Technische Universität München

\section{Background:}

Crohn's disease (CD) is characterized by relapsing intestinal inflammation leading to severe diarrhea, weight loss and abdominal pain. The anti-inflammatory protein Annexin A1 (AnxA1) has been linked to inflammatory processes in the gut. It exerts anti-inflammatory effects by suppression of leukocyte activation and transmigration, and exhibits anti-inflammatory properties in various inflammation models. The exact expression pattern of AnxA1 in patients with CD has not been examined yet. This project aims to investigate AnxA1 expression patterns in the inflamed intestine of CD patients and correlate AnxA1 expression capacity in the inflamed tissue to disease severity.

\section{Materials and methods:}

Surgical specimens of patients with CD undergoing intestinal surgery between 2003 and 2015 at our institution were examined. Immunohistochemistry (IHC) was performed to delineate AnxA1 expression in the inflamed intestine. Those with pronounced AnxA1 protein expression were included in further analysis by qPCR. RNA was isolated from the inflamed and non-inflamed regions detected by a pathology specialist. AnxA1 mRNA expression ratio of the inflamed to non-inflamed areas was calculated and defined as expression capacity of the inflamed tissue. Depending on their expression capacity, patients were divided into two groups (AnxA1-low and AnxA1-high). Correlation of AnxA1 expression capacity to clinical features was performed.

\section{Results:}

In IHC, AnxA1 protein expression was correlating to the severity of inflammation in the intestinal tissue. In qPCR, AnxA1 mRNA expression was upregulated 20 -fold in inflamed to non-inflamed tissue $(p<0.03)$. Patients with higher intrinsic AnxA1 expression capacity ( $>20$, AnxA1-high group) had significantly less severe inflammation ( $14.3 \%$ vs. $57.1 \%, p<0.049)$ compared to AnxA1-low group (expression capacity < 1). Furthermore, AnxA1-high group had significantly more locally restricted disease $(100 \%$ vs $57.1 \%, p<0.007)$, more stricturating disease $(85.7 \%$ vs. $28.6 \%, p<0.004)$ and less preoperative steroid therapy $(42.9 \%$ vs. $78.6 \%, p<0.030)$.

\section{Conclusion:}

AnxA1 expression resembles intestinal inflammation in patients with Crohn's disease on protein and RNA level. Patients with higher intrinsic AnxA1 expression capacity of the inflamed tissue seem to have a milder course of disease with milder inflammation, more located disease, less penetrating disease and less need for preoperative steroid therapy. 


\section{Picture:}
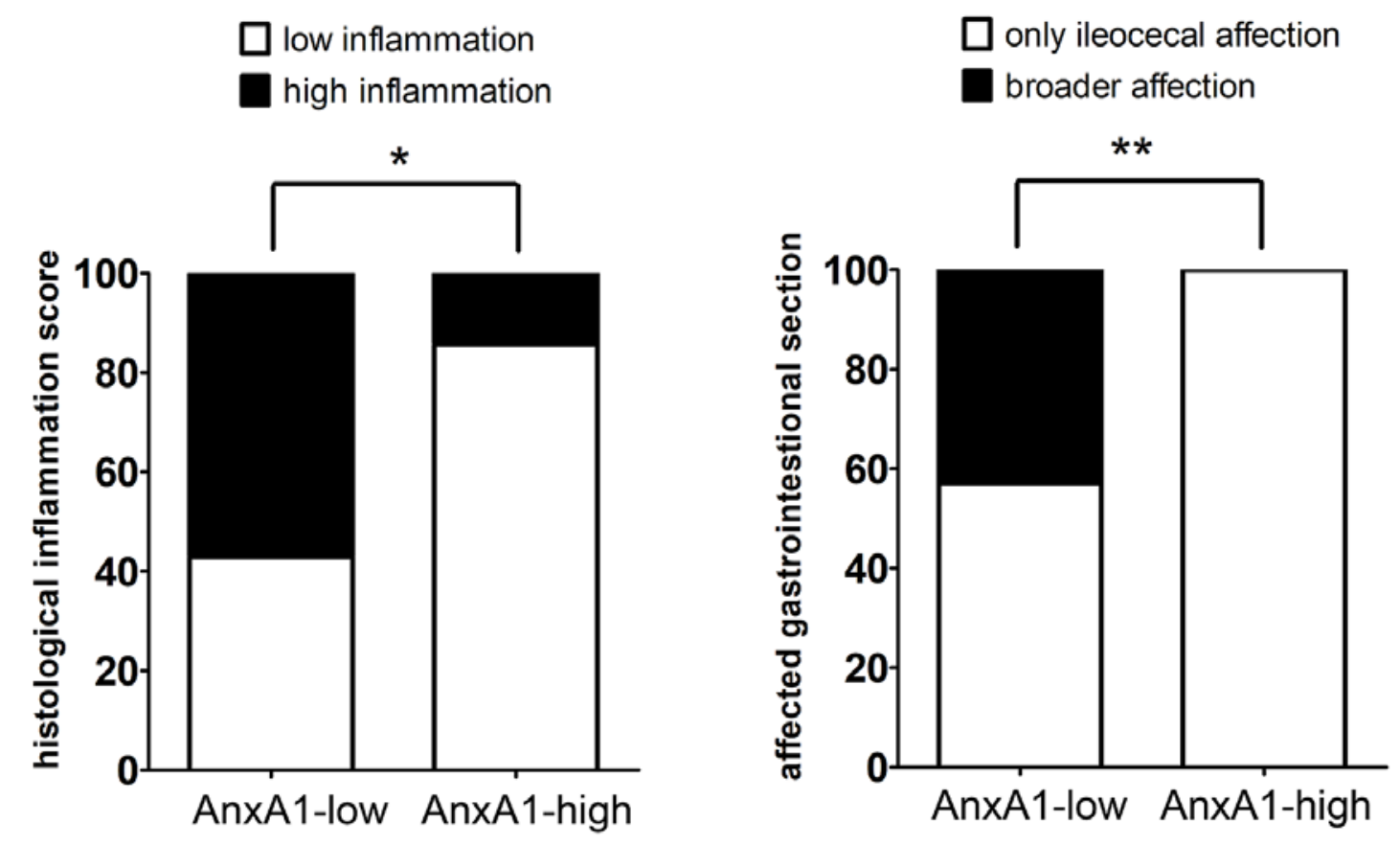

stricturating disease

non-stricturating disease

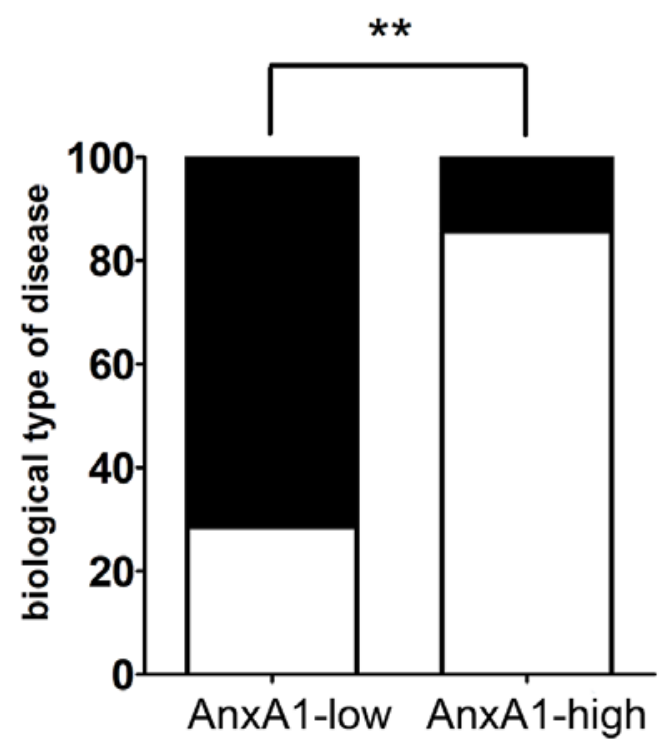

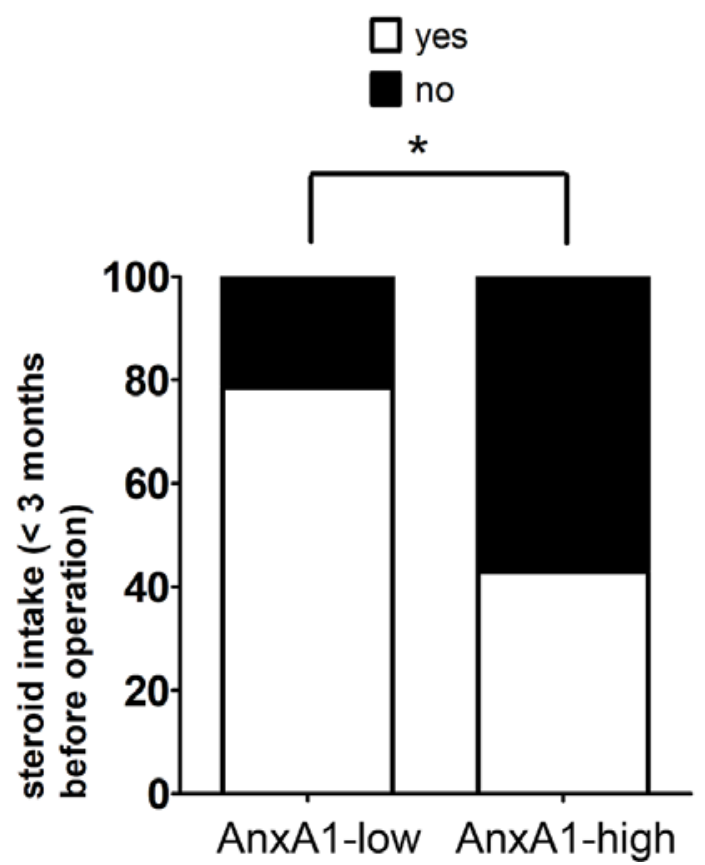

The effect of AnxA1 expression capacity on severity of inflammation, location and type of disease and preoperative steroid treatment 
Unplanned Readmission within 90 days After Oncologic Pancreatic Surgery: Causes, Causality and Treatment Pattern

(Abstract ID: 951)

A. Imamovic ${ }^{1}$, T. Niernberger ${ }^{2}$, S. Gabor ${ }^{2}$, S. Sauseng ${ }^{1}, H$. Rabl $^{3}$

${ }^{1}$ LKH-Universitätsklinikum Graz

${ }^{2}$ Burgenländische Krankenanstalten A.ö. Krankenhaus Oberwart

${ }^{3}$ Landeskrankenhaus Hochsteiermark, Standort Leoben

\section{Background:}

Readmission rates have been targeted as a quality metric for patient's condition, surgical performance and cost control. The readmission rate within 30 days is about $19 \%$.

\section{Materials and methods:}

Our retrospective analysis included 143 patients (m:f $=76: 67$ ) who were underwent a classical Whippel procedure, PPPD and left sided resection with readmission within 90 days.

\section{Results:}

Readmission rate was $25 \%$ ( $36 / 143$ pts.). Operation associated readmission rate was 10,5\% (15/36 pst.) and included hepatic abscess (4/15 pts.), persistend diarrhoea (3/15 pts.), portal vein thrombosis (3/15 pts.), retroperitoneal abscess (1/15 pts.), infected pseudocyst (1/15 pts.), arrosion bleeding (1/15 pts.), subhepatic hematoma (1/15 pts.) and stump leakage after left sided resection (1/15 pts.).

Redo-surgery was done in 3 patients (2\%), interventional radiologic / endoscopic treatment in $2(1,5 \%)$ and conservative treatment in 10 patients $(7 \%)$.

Non surgical associated readmission rate was $14,7 \%$ (21 pts.) due to ascites, pulmonary embolism, pneumonia and reduced general health.

\section{Conclusion:}

The low need for interventions whether surgical or radiological / endoscopical was $3,4 \%$ and was successful in each case 


\section{„Safety first!?"- the (negative) impact of global health policy on surgical primary care}

(Abstract ID: 106)

I. Metelmann ${ }^{1}$, A. Busemann ${ }^{2}$

${ }^{1}$ Universitätsklinikum Leipzig

${ }^{2}$ Universitätsmedizin Greifswald

\section{Background:}

There is an alarming inequality in global surgical care with a severe undersupply in low- and middleincome countries. Reasons for that are various while most studies emphasize the widespread misperception, that a global improvement of surgical care would be cost ineffective. However, the aspects of health security remain rather unmentioned in the ongoing scientific discussion. The development of a resilient system for surgical care asks for long-term horizontal health interventions. The outbreak of Ebola in Western Africa in 2014 shed new light on the significance of health security on the international health agenda.

\section{Materials and methods:}

The political science concept of securitization by the Copenhagen School is used to show how the Ebola crisis has been securitised and serves as the theoretical framework for a case study of the surgical care in Sierra Leone before, during and after the outbreak of Ebola in 2014.

\section{Results:}

Surgical care in Sierra Leone declined massively during the 2014 Ebola crisis. This was driven by widely communicated fear in the health sector, insufficient employee protection and a focus on vertical crisis management interventions.

\section{Conclusion:}

The significance of surgery on the global health agenda is relevantly compromised by the current health security policy. The peaking use of vertical health interventions in times of health crisis threatens the sustainable development of surgical care in low- and middle-income countries. 


\section{A full-size adipose pig model for bariatric interventions}

(Abstract ID: 1050)

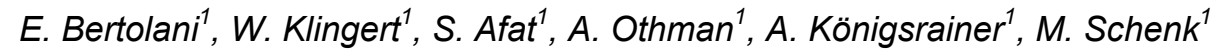

${ }^{1}$ Universitätsklinikum Tübingen

\section{Background:}

The suitability of available mini-pig models of adiposity for bariatric interventions is limited due to anatomical restrictions (e.g. stomach size).

Weight-loss studies in landrace pigs affecting mainly muscle mass are unethical.

Objectives

Development of an anatomical adipose pig model

\section{Materials and methods:}

Two Mangalitza breed pigs were used.

A total of $4.5 \%$ of the body weight was fed twice daily.

First feeding was after weighing in the morning. Second feeding time was approximately 8-10 hours after the first. Each feeding consists of fattening flour, equal amount of wheat (at least $250 \mathrm{~g} / \mathrm{kg}$ feed weight), rapeseed oil (at least $70 \mathrm{~g} / \mathrm{kg}$ feed weight), clarified butter (at least $150 \mathrm{~g} / \mathrm{kg}$ feed weight), soybean oil (50 g / kg feed weight), fructose $(50 \mathrm{~g} / \mathrm{kg}$ feed), dextrose $(50 \mathrm{~g} / \mathrm{kg}$ feed). This was mixed with enough water to a pulp. As feed intake decreased, food was varied in flavor by adding mashed potato powder or apple juice.

A longitudinal determination of the fat mass increase was performed on the animals using CT imaging (figure 2).

\section{Results:}

The weight of the animals increased linearly from $60 \mathrm{~kg}$ to $160 \mathrm{~kg}$ over a period of 30 weeks.

The quantification of total adipose tissue showed an increase in visceral and subcutaneous adipose

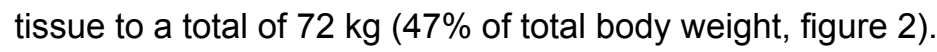

In behavior analysis and clinical chemistry no adverse effects were noted.

\section{Conclusion:}

Through targeted feeding, an anatomically obese pig model can be achieved. 


\section{Picture:}

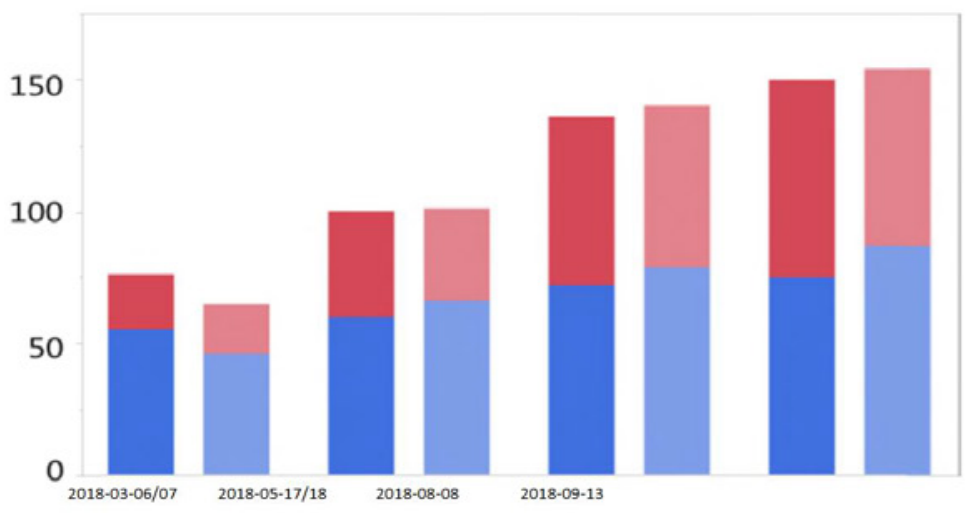

Figure 1: quantification of total tissue (red = weight of adipose tissue; blue = weight of other tissue)

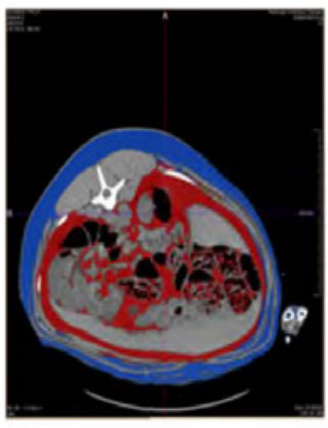

2018-03-06/07
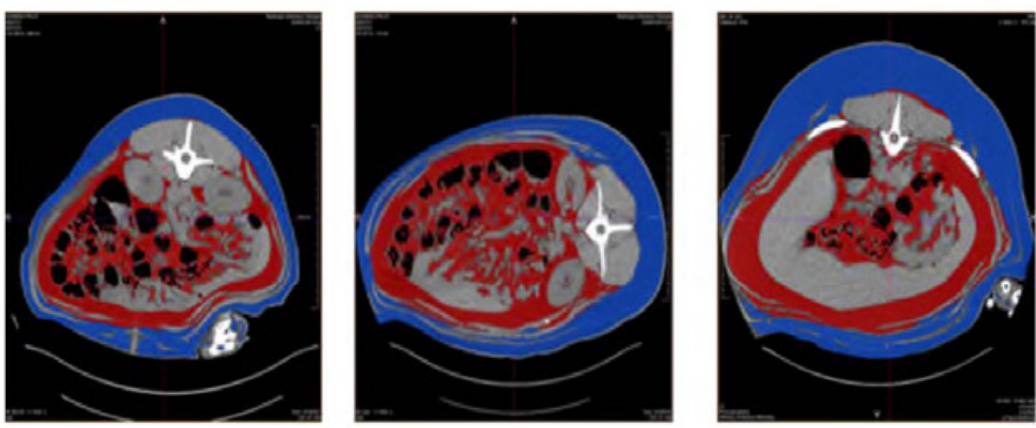

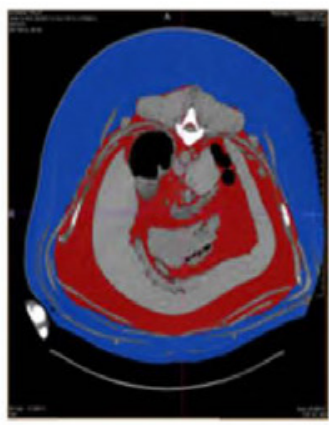

2018-09-13

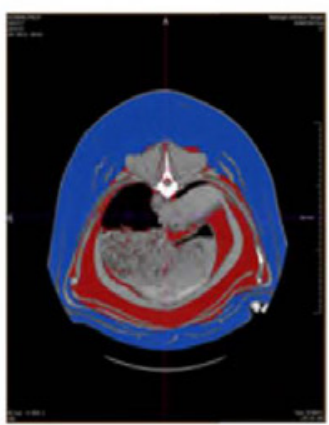

Figure 2: quantification of adipose tissue (red: visceral fat, blue: subcutaneous fat) 
New era in colon surgery: Establishing mesocolic lymph node mapping via indocyanine green (ICG)

(Abstract ID: 460)

\author{
M. Kalandarishvili ${ }^{1}$, M. Kaspari ${ }^{1}$, G. Winde ${ }^{1}$, E. W. Kolbe ${ }^{1}$ \\ ${ }^{1}$ Klinikum Herford
}

\title{
Background:
}

Definitive treatment of colon cancer includes radical resection and in defined nodal positive cases adjuvant chemotherapy. Localisation and penetration depth of the tumour defines the extent of the operation. Lymphadenectomy in this procedure is standardized, but in some cases the degree of this resection is debatable. Furthermore, the role of sentinel lymph node (SLN) mapping in colon cancer is not clarified so far.

\section{Materials and methods:}

Since April 2019 eight patients with colon cancer underwent radical laparoscopic assisted resection. After tumour identification due to palpation or after detecting the color coding marking of the preoperative colonoscopy, 10-20 mg of diluted indocyanine green (ICG) was injected into the colonic wall nearby the tumour location. Indocyanine green is distributed via lymphatic tissue and accumulate at the lymph nodes. ICG is detected via a specialized fluorescence system (TC300, TCD201, TH102, 20133720, Karl Storz, Tuttlingen, Germany) exciting the ICG and assessing lymphatics in real time. The SLN is identified by a clear bright green fluorescence and marked using a metal clip to facilitate the further histopathological examinations.

\section{Results:}

After applying ICG the SLN was identified 10-20 minutes later, 50-70 minutes after ICG injection the whole lymphatic drainage is visible. The SLN identified via ICG fluorescence could be verified histologically in $6 / 8$ cases.

\section{Conclusion:}

Detecting and resection of SLN in colon cancer is not established so far. According to our results it is feasible to retrace the propagation track of the colon cancer via lymphatic tissue spreading in real time using ICG. The SLN detected by ICG illumination is confirmed by histological elaboration in most cases. So additional questions could be answered in further studies: Is there a correlation between the data gained by SLN/ICG staining and the well-known anatomic borders of lymphatic tissue related to the arterial vascular system of the colon? Are there histologically nodal positive cases, whereas the SNL is identified but not infiltrated by colon cancer cells, especially in earlier pT-stages? Is there a possibility to have nodal positive cases without a positive SNL staining, e.g. by lymphatic bypassing of cancer cells? Is the SNL comparable to the central lymph node in the anatomic radical central resection site.

So far, ICG imaging of the lymphatic system in colon cancer is at least an appropriate method to secure the extent of performing a radical mesocolic excision and particularly identifying the deepest layer of the central lymph nodes. 